\title{
Males deploy multifaceted strategies and hijack longevity pathways to induce premature demise of the opposite sex
}

Lauren N. Booth ${ }^{1}$, Cheng Shi ${ }^{2,3}$, Cindy Tantilert ${ }^{1}$, Robin W. Yeo ${ }^{1}$, Katja Hebestreit ${ }^{1,4}$, Cecilia N. Hollenhorst $^{1}$, Coleen T. Murphy ${ }^{2,3, *}$, Anne Brunet ${ }^{1,5, *}$

${ }^{1}$ Department of Genetics, Stanford University School of Medicine

${ }^{2}$ Department of Molecular Biology, Princeton University

${ }^{3}$ LSI Genomics, Princeton University

${ }^{4}$ Present address: BioMarin Pharmaceutical, San Rafael, CA

${ }^{5}$ Glenn Laboratories for the Biology of Aging and Stanford University

* Co-corresponding authors

ctmurphy@princeton.edu

abrunet1@stanford.edu 


\begin{abstract}
Interactions between the sexes negatively impact health in many species, including mammals ${ }^{1-9}$. In mice, sexual interactions induce weight gain and shorten lifespan in females, independent of fertilization, ${ }^{6,9}$. In Caenorhabditis, males shorten the lifespan of the opposite sex (females or hermaphrodites $)^{1-3,8}$. However, the mechanisms underlying the negative influence of males on lifespan - and their overlap with known longevity pathways - are still largely unknown. Here, we use transcriptomic profiling and targeted screens to systematically uncover new conserved genes involved in male-induced demise. Interestingly, deficiency of these genes individually, and especially in combination, induces strong protection, highlighting the benefit of combining interventions to extend lifespan. Some genes (e.g. acbp-3, col-43) only extend hermaphrodite lifespan when knocked-down in the presence of males, suggesting specific protective mechanisms against male-induced demise. However, we also uncover two previously unknown longevity genes (sri-40 and delm-2) that, when knocked-down, extend hermaphrodite lifespan both with and without males, which points to new broad mechanisms of resistance. In sharp contrast, many classical long-lived mutants are actually short-lived in the presence of males, suggesting that males hijack and suppress known longevity pathways. This systematic analysis reveals striking differences in longevity in single sex versus mixed sex environments and uncovers the elaborate network of functional regulation elicited by sexual interactions, which could extend to other species.
\end{abstract}

\title{
Main
}

Sexual interactions influence organismal health independently of reproduction in nematodes, flies, and mammals ${ }^{1-9}$. However, the impact of sexual interactions on health is still largely uncharacterized, in large part because most experiments are conducted in single-sex environments. Because of its short lifespan, C. elegans is an ideal model organism to systematically examine how sexual interactions affect longevity. Sexual interactions with males shorten the lifespan of the opposite sex (females or hermaphrodites) in Caenorhabditis ${ }^{1-3,8}$, and this phenomenon has been shown to involve components from both sexes. Indeed, males promote the premature death of hermaphrodites using male sperm and seminal fluid during mating ${ }^{2}$ as well as male pheromones and secreted compounds ${ }^{1,10}$ (especially when large numbers of males are present $^{11}$ ). In hermaphrodites, several molecular and cellular pathways have been found to mediate aspects of 
male-induced demise, including transcription factors (e.g. FOXO/DAF-16 and TFEB/HLH-30) 2,12, chromatin regulators (e.g. KDM6A/UTX-1) ${ }^{1}$, insulin ligands (e.g. INS-11 and INS-7) ${ }^{1,2,12}$, and even self-sperm itself ${ }^{12,13}$. However, a systematic investigation of the pathways driving the negative impact of males on lifespan, and how they overlap with known longevity pathways, is still missing.

To determine in a systematic manner the impact of sexual interactions with males on the opposite sex in C. elegans, we performed RNA sequencing (RNA-seq) of young (day three of life) and middle-aged (day seven of life) hermaphrodites cultured either in the presence or absence of males (Fig. 1a, see Materials and Methods). The males were present for a brief exposure (one day) or for a long exposure (five consecutive days) (Fig. 1a). To eliminate possible effects of sexual interactions during development ${ }^{10,14,15}$ and avoid confounds from embryo transcripts, we initiated exposure to males at the onset of adulthood in sterile $\left(\mathrm{glp}-1[\mathrm{e} 2144]^{16}\right)$ hermaphrodites (Fig. 1a). Male-induced demise occurs robustly and reproducibly under these conditions (Fig. 1b) ${ }^{1,2}$.

Principal component analysis separated the transcriptomes of the samples based on age (Fig. 1c, Extended Data Fig. 1a) and exposure to males, especially after longer exposure (Fig. 1d-e). Prolonged exposure to males resulted in a marked increase in the degree and number of gene expression changes compared to the brief exposure to males (Fig. 1f). The genes that were induced in hermaphrodites following a brief (one day) or long (five days) interaction with males partially overlap (e.g. ins-11, sri-40, acbp-3) (Fig. 1g). Importantly, many of the gene expression changes that we observed in sterile, $g l p-1$ hermaphrodites following sexual interactions with males were also observed in wild-type and fertile hermaphrodites (Extended Data Fig. 1c-e) ${ }^{13}$. Genes upregulated in response to males were enriched for lipid metabolism and transport, collagens, and stress response (detoxification) gene categories (Fig. 1h, Extended Data Fig. 1e). Thus, males induce transcriptional changes in hermaphrodites, with a greater number and magnitude of changes in response to long interactions.

As both brief and prolonged exposure to males shortens hermaphrodite lifespan ${ }^{1,2}$ (Supplementary Table 1), genes that are induced in both conditions might be more functionally relevant to elicit demise and we focused on these for the remainder of the study. To understand the role of these genes in the male-induced demise of hermaphrodites, we performed a targeted, RNAibased screen (Fig. 1i and Extended Data Fig. 2). Using wild-type, fertile hermaphrodites, we 
individually knocked-down male-induced hermaphrodite genes identified through RNA-seq and measured hermaphrodite lifespan in the presence and absence of males. Our screen newly identified ten genes (out of 26 genes tested) that, when knocked-down, partially protected hermaphrodites from male-induced demise (acbp-3, acox-1.3, col-43,col-125, cyp-25A3, delm-2, lpr-6, sre-28, sri-40, and wrt-10) (Fig. 1i and Extended Data Fig. 2, Supplementary Table 1, see also Fig. 2-4 for further analysis of these genes). Together, these results show striking transcriptional effects of sexual interactions on the opposite sex and identify a set of genes that are functionally important regulators of lifespan in response to sexual interactions.

We wondered whether the genes we identified as functional mediators of the negative impact of males on lifespan are induced differently by the various male components known to contribute to premature demise (male sperm, seminal fluid, and pheromones ${ }^{1,2,11}$ ), as this could lead to the development of combined interventions against male-induced demise. To this end, we measured gene expression in young (day 4-5 of life) hermaphrodites following one day of mating with both wild-type males and sperm-less (fer-6[hc6 ${ }^{17}$ ) males (Fig. 2a,b and Extended Data Fig. 3a-d) (thereby distinguishing male sperm from seminal fluid), or on male-conditioned plates for five days (isolating male pheromones) (Fig. 2c). Interestingly, each male component elicited a distinct transcriptional response from hermaphrodites (Extended Data Fig. 3e), consistent with the different physiological changes (e.g. fat loss and body shrinking) observed in response to these male components ${ }^{2,11}$.

The subset of genes induced by male sperm (i.e. expressed in response to mating with wildtype but not sperm-deficient males) were enriched for metabolism genes, and included an acylCoA binding protein gene ( $A C B D 4,5 / a c b p-3)$ and an insulin peptide involved in innate immunity $\left(\right.$ ins-11) ${ }^{18}$ (Fig. 2a). RNAi knock-down of the male sperm-dependent genes acbp-3 (Fig. 2d) and ins- $11^{1}$ (as well as other male sperm induced genes) each protected hermaphrodites from maleinduced demise (increasing median lifespan by $14-25 \%$, see Supplementary Table 1). Thus, acbp3 is a novel gene involved in premature demise via male sperm.

The subset of genes that were likely induced by male seminal fluid (i.e. expressed both in response to mating with wild-type males as well as fer-6(hc6) males that cannot make sperm but still transfer seminal fluid ${ }^{17}$ ) were enriched for sodium channel proteins and include the degenerinlike ion channel genes $A S I C 1-5 /$ delm-2 and $a c d-1^{19,20}$ (Fig. 2b). RNAi knock-down of delm-2 
(which likely also targets delm-1 and acd-1 paralogs, Extended Data Fig. 4) and acd-1 (and other seminal fluid induced genes) each partially protected hermaphrodites from male-induced demise (8-29\% increase in median lifespan, see Supplementary Table 1) (Fig. 2e and Fig. 4d). Thus, delm2 and $a c d-1$ (and possibly their paralog delm-1), as well as sri-40 play functional roles in premature hermaphrodite demise via male seminal fluid.

Male-secreted compounds, including pheromones, can shorten hermaphrodite lifespan in the absence of mating ${ }^{1,10,11}$. To identify genes involved in the response of male-secreted compounds (including pheromones), we exposed young, wild-type hermaphrodites to maleconditioned plates and performed a microarray on the hermaphrodites following five days of exposure (Fig. 2c). Genes induced by male-conditioned plates include a solute carrier/transporter gene (hmit-1.1). Interestingly, RNAi knock-down of hmit-1.1 (and other male-conditioned plate induced genes) protected hermaphrodites from the lifespan shortening effect of male-conditioned plates (up to a 17\% increase in median lifespan, see Supplementary Table 1) (Fig. 2f and Extended Data Fig. 5). Thus, hmit-1.1 and C33G8.3 are novel regulators of hermaphrodite demise induced by male-secreted compounds, including male pheromones.

Interestingly, many of the genes induced by the different male components are conserved between $C$. elegans and humans. Some of the human genes are expressed in the same tissues as their C. elegans orthologs (Fig. 2g-i and Extended Data Fig. 6), where they could initiate a conserved response to sexual interactions. Collectively, these findings show that male sperm, seminal fluid, and pheromones induce the expression of different subsets of novel and conserved genes that functionally contribute to premature demise in the opposite sex.

The observation that different genetic pathways mediate the demise induced by male sperm, seminal fluid, and pheromones raises the possibility that modulating these genes in combination could be more effective to counter male-induced demise (Fig. 3a). To test this, we performed lifespan assays in which we combined losses of gene function. We assessed the role of the gene delm-2 (induced by male seminal fluid) in combination with acbp-3 (induced by male sperm) (Fig. $3 b)$. While knock-down of each gene individually resulted in a partial protection from maleinduced demise, knock-down of delm-2 and $a c b p-3$ together resulted in additive protection against male-induced demise (11-31\% increase in median lifespan compared to a single gene knock-down, see Supplementary Table 1) (Fig. 3b). Interestingly, knock-down of delm-2 and acbp-3 in 
combination fully protected hermaphrodites from the lifespan shortening impact of sexual interactions with males $(43-50 \%$ increase in median lifespan compared to control RNAi, see Supplementary Table 1) (Fig. 3c), and these hermaphrodites lived approximately the same lifespan as hermaphrodites in the absence of males (Fig. 3c, Supplementary Table 1). Thus, a combined loss of function in the male sperm and male seminal fluid pathways improves protection from male-induced demise. Analysis of the regulatory regions of the genes induced by parallel male strategies revealed key transcription factor binding sites (Fig. 3d), with some of them being shared among different pathways (e.g. PQM-1 ${ }^{12}$, PPARD/NHR-28, and conserved PBX3,4/CEH-60) (Fig. 3d, Extended Data Fig. 7). Together, these results suggest that targeting the different strategies induced by males in combination (using multiple genes and perhaps common transcription factors) is more effective to counter male-induced demise than targeting a single strategy.

We next wondered whether genes that mediate male-induced demise are specific to the presence of males or whether they have a more general effect on lifespan, and how this compares to traditional longevity mutants (Fig. 4a). Several of the male-induced genes we identified specifically extended lifespan when knocked-down only in the presence of males (e.g. acbp-3, col43), and did not confer a longer hermaphrodite lifespan in the absence of males (Fig. 4a-e). These genes represent pathways that are specifically involved in regulating longevity in a mixed sex environment.

Other genes lead to lifespan extension when knocked-down both in absence and presence of males. We previously found that $u t x-1$ RNAi knock-down extends hermaphrodite lifespan in the absence of males ${ }^{21,22}$ and protects hermaphrodites from male-induced demise ${ }^{1}$. Here, we identify three additional genetic interventions that protected from male-induced demise and promoted longevity in the absence of males: RNAi knock-down of delm-2 or sri-40 individually (Fig. 4a, $\mathrm{f}$, g) and knock-down of delm-2 and $a c b p-3$ in combination (Fig. 4h). These are genes that can regulate longevity in both a single and mixed sex environment.

In sharp contrast, traditional long-lived mutant hermaphrodites (daf-2[e1370] ${ }^{23,24}$, glp$1[e 2144]^{25}$ ) are hypersensitive to male-induced demise ${ }^{1,2}$. Similar to daf-2 mutants, we find that long-lived mitochondria mutants (e.g. $i s p-1^{26}, n u o-6^{27}$ ) are also hypersensitive to the presence of males, as their lifespan extension is smaller (or undetectable) in the presence of males compared 
to the absence of males (Fig. 4a, 4i, j). This may be due to males "hijacking" traditional longevity genes and pathways to repress them, possibly through inhibition of the FOXO/DAF-16 transcription factor ${ }^{2,23-25,28}$. These genes represent pathways that are strongly involved in regulating lifespan in a single sex environment, but not in a mixed sex environment.

Together, these results show that males impinge on the health of the opposite sex in different ways - highly specific pathways (e.g. acbp-3), more general health pathways (e.g. delm-2), and the "hijacking" of conserved longevity pathways (Fig. 4k and Extended Data Fig. 8). These findings also reveal that the context in which an individual lives (for example, with or without mates) can drastically impact the effect of a longevity mutation.

Here, we have identified genes that are not only regulated by but are also functionally important for the response of hermaphrodites to sexual interactions (male-induced demise). By investigating these genes through different "lenses" we have found that they fall into different functional categories both in terms of the male signal to which they respond (male sperm, seminal fluid, or pheromones) and the specificity of their regulation of lifespan (specific to male-induced demise versus broad longevity regulation). Many of these genes are conserved in mammals, and they could also play a role in lifespan and health in other species, including humans.

The placement of the male-induced demise genes in different pathways allowed us to predict which combination of strategies could lead to greater protection from male-induced demiseknock-down of independent male-induced demise pathways in combination has an additive effect towards protecting hermaphrodites against males. In fact, knock-down of delm-2 and acbp-3 in combination extended the lifespan of hermaphrodites in the presence of males to the lifespan of hermaphrodites in the absence of males. This is important because it could help identify additional combinations of strategies to more robustly extend lifespan.

Our analysis identifies genes whose knock-down can extend lifespan both in the absence and presence of males (e.g. sri-40 and delm-2) and that have been missed by previous genetic screens. Thus, elucidating the detrimental effects of sexual interactions on health can also identify pathways that regulate health more generally. These newly-identified regulators of longevity have promise to protect individuals from a range of different stressors, unlike the specific regulators of maleinduced demise we have identified (e.g. $a c b p-3$ and col-43). In contrast, several classical longevity mutants are, in fact, short-lived (hypersensitive) in the presence of males. These results suggest 
that males hijack components of traditional longevity pathways and that long-lived mutants may have an Achilles' heel - their susceptibility to the opposite sex. Sexual interactions may therefore represent a particularly potent biological force distinct from other types of stresses. Our study also reveals that targeting genes in a mixed vs. a single sex environment can have different outcomes on lifespan, which has important implications for health. 


\section{Acknowledgements}

We thank Anne Villeneuve, Miriam Goodman, and Laura Bianchi for helpful discussions. Thank you to all the members of the Brunet lab, particularly Jingxun Chen, Salah Mahmoudi, Jason Miklas, and Katharina Papsdorf for helpful feedback and discussion. Thank you to the members of the Murphy lab for valuable feedback. The Stanford Functional Genomics Facility performed the sequencing of the RNA-seq libraries. We thank the Caenorhabditis Genetics Center (funded by NIH grant P40 OD010440), Miriam Goodman, Laura Bianchi, and Man-Wah Tan for providing $C$. elegans strains used in this study and WormBase. This work was funded by R01AG054201 (AB), HHMI-Simons Faculty Scholars Program (CTM), Glenn Foundation for Medical Research (CTM), Genentech Foundation Predoctoral Fellowship (RWY), the Helen Hay Whitney Foundation (LNB), and NIH K99 AG051738 (LNB).

\section{Author Contributions}

This study was designed by LNB, CS, CTM, and AB. LNB performed the RNA-seq experiments and data were analyzed by LNB and KH. Microarrays were performed by CS and analyzed by CS, LNB, and RWY. All LNB and KH-written code was independently checked by RWY. Lifespan assays were performed and analyzed by LNB, CS, and CT (see Supplementary Table 1 for the assays that were performed by each researcher). $\mathrm{CNH}$ performed assay validation. The figures were prepared by LNB with feedback from AB, CTM, and CS. The original manuscript draft was written by LNB with advice and editing from AB, CTM, and CS.

\section{Competing Interests}

The authors declare no competing interests.

\section{Data Availability}

All RNA-seq reads are available on NCBI Sequence Read Archive (PRJNA642294). The results from the microarrays are available at http://puma.princeton.edu (see Materials and Methods). The complete list of all lifespan assays (including statistics and number of animals) is presented in Supplementary Table 1 and the results of our RNA-seq and microarray analyses are presented in Supplementary Tables 2 to 10 . 
bioRxiv preprint doi: https://doi.org/10.1101/2020.06.30.181008; this version posted July 1, 2020. The copyright holder for this preprint (which was not certified by peer review) is the author/funder, who has granted bioRxiv a license to display the preprint in perpetuity. It is made available under aCC-BY-NC-ND 4.0 International license.

\section{Code Availability}

All code is available on GitHub (https://github.com/brunetlab/Booth-et-al.-2020). 


\section{Materials and Methods}

\section{Worm strains and maintenance}

All C. elegans wild-type and mutant strains used in this study are listed below. All strains were maintained on Nematode Growth Media (NGM) plates with $50 \mu \mathrm{g} / \mathrm{mL}$ streptomycin (Gibco) and a lawn of OP50-1 bacteria (a gift from M.-W. Tan) from stationary phase cultures. Nematodes were grown at $20^{\circ} \mathrm{C}$, with the exception of temperature-sensitive mutants $(\mathrm{glp}$ $1[e 2144]$ and $[e 2141])$, which were maintained at $15^{\circ} \mathrm{C}$ (permissive temperature). When temperature sensitive mutants were used for assays, they were grown at the restrictive temperature $\left(25^{\circ} \mathrm{C}\right)$. The genotype of strains was verified by genotyping PCR and Sanger sequencing and the strains were backcrossed three times into our laboratory's N2 strain (in addition to the backcrossing that was performed when the mutants were initially isolated).

\begin{tabular}{|l|l|l|}
\hline C. elegans Strain & Genotype & Source \\
\hline N2 & wild-type & Dr. Man-Wah Tan and CGC \\
\hline CF1903 & glp-1(e2144) III & CGC \\
\hline CB4037 & glp-1(e2141) III & CGC \\
\hline CB1467 & him-5(e1467) $V$ & CGC \\
\hline BA6 & fer-6(hc6) I & CGC \\
\hline CB4108 & fog-2(q71) $V$ & CGC \\
\hline RB1523 & delm-2(ok1822) I & CGC and CDMC \\
\hline RB1177 & delm-1(ok1226) IV & CGC and CDMC \\
\hline BLC100 & delm-1(ok1226) IV; & Dr. Laura Bianchi \\
& delm-2(ok1822) I & \\
\hline ZB90 & $a c d-1(b z 90) I$ & Dr. Laura Bianchi \\
\hline CB1611 & $m e c-4(e 1611) X$ & Dr. Miriam Goodman \\
\hline MQ1333 & nuo-6(qm200) I & CGC \\
\hline MQ887 & isp-1(qm150) IV & CGC \\
\hline CB4876 & $c l k-1(e 2519) I I I$ & CGC \\
\hline
\end{tabular}

*CGC is the Caenorhabditis Genetics Center

${ }^{*} \mathrm{CDMC}$ is the C. elegans Deletion Mutant Consortium ${ }^{29}$

\section{RNA-seq}

To better understand how males induce premature hermaphrodite demise, we characterized the transcriptomes of sterile $g l p-1($ e2144) hermaphrodites that have a shortened lifespan after 
interacting with wild-type males for one day or five days to those that never interacted with males.

Individuals were age-synchronized using a brief, 3-4 hour egg lay (see "Lifespan Assays" below) on $10 \mathrm{~cm}$ NGM plates seeded with OP50-1 bacteria and grown at $25^{\circ} \mathrm{C}$ during development and adulthood. The day of the egg lay is considered day 0 of life. For the longer (five day) exposure to males, $75 \mathrm{glp}-1(\mathrm{e} 2144)$ hermaphrodites were placed onto a $10 \mathrm{~cm}$ NGM plates seeded with OP50-1 bacteria and with 75 young (day 3-5 of life) WT males starting on day 2 of life (young adults) until day 7 of life. At day 5 of life, the hermaphrodites were moved to fresh plates and the males were replaced with new, young WT males. For samples in which hermaphrodites interacted with males for a single day, $75 \mathrm{glp}-1(\mathrm{e} 2144)$ hermaphrodites were placed onto a 10cm NGM plates seeded with OP50-1 bacteria and 75 young (day 3-5 of life) WT males were added starting on either day 2 of life (young adults) or on day 6 of life. Hermaphrodites that interacted with males starting on day 6 of life were maintained on $10 \mathrm{~cm}$ NGM plates with OP50-1 at a density of 150 hermaphrodites starting from day 2 of life. At the same time, hermaphrodites from the same cohort of age-synchronized glp-1(e2144) individuals were also placed on fresh plates without males $(150$ hermaphrodites per $10 \mathrm{~cm}$ plate to maintain a similar density). After one or five days, 75 hermaphrodites from each condition (individuals that either interacted with males or never interacted with males) were collected by removing either 75 hermaphrodites (for the "no males" condition) or the 75 males from the plates. For each sample, these remaining 75 hermaphrodites were immediately washed three times with ice cold M9 buffer $\left(22 \mathrm{mM} \mathrm{KH}_{2} \mathrm{PO}_{4}, 42 \mathrm{mM} \mathrm{Na}_{2} \mathrm{PO}_{4}, 86 \mathrm{mM} \mathrm{NaCl}\right.$, and $\left.1 \mathrm{mM} \mathrm{MgSO}_{4}\right)$ and the worm pellets were flash frozen in liquid nitrogen. In parallel with the RNA-seq sample collection, $g l p$ 1(e2144) hermaphrodites and WT males form the same batches of age-synchronization as their corresponding RNA-seq samples were used to measure hermaphrodite lifespan as described in the section "Lifespan Assays" (see Supplementary Table 1).

To determine the contribution of male transcripts to the $g l p-1($ e2144) samples, we also isolated and prepared for RNA-sequencing the population of WT males that were on the RNAseq replicates E-H plates with the day 6-7 of life, single day sexual interaction condition. These young male worms (not synchronized) were washed with ice cold M9 buffer and flash frozen as described above. 
RNA was extracted from the flash frozen worm pellets (approximately 75 whole worms per sample) with $500 \mu \mathrm{L}$ Trizol and $200 \mu \mathrm{L}$ chloroform followed by $250 \mu \mathrm{L}$ phenol and $200 \mu \mathrm{L}$ chloroform extractions and finally, an isopropanol precipitation. Remaining DNA was degraded with DNaseI (Promega) and the RNA cleaned with a sodium acetate and ethanol precipitation. RNA quality was measured using Nanodrop spectrophotometry and the Agilent BioAnalyzer Total RNA Nano chip and kit. mRNA enriched cDNA was prepared using 10ng of total RNA (quantified by Nanodrop spectrophotometry) and the Takara SMART-seq v4 Ultra Low Input RNA kit, with 8 rounds of amplification. Paired-end libraries were made using the Nextera XT DNA library prep kit (Illumina) with 1ng of cDNA (quantified using the Qubit dsDNA High Sensitivity reagents, Invitrogen) and barcoded using the Nextera XT Index Kit v2 (Illumina). Libraries were purified with $30 \mu \mathrm{L}$ AMPure XP beads (Beckman Coulter) as directed in the Nextera XT kit. Library quality and quantity were assessed using the Agilent Bioanalyxer High Sensitivity DNA Assay. The libraries for biological replicates A-D were prepared together and pooled and sequenced on a single Illumina NextSeq run and the libraries for biological replicates E-H were prepared together and pooled and sequenced on two Illumina NextSeq runs. Pairedend, 75 base pair sequencing was performed. All RNA-seq reads are publicly available through NCBI Sequence Read Archive (BioProject PRJNA642294).

\section{RNA-seq analysis}

RNA-seq reads were aligned to the WBcel235 genome and gene read counts were calculated using STAR (version 2.5.4a) ${ }^{30}$. Low-coverage genes that had less than one read count per million mapped reads in less than three samples were filtered out. After filtering of low-coverage genes, 16,706 genes remained (prior to removal of male-enriched genes, see below) with read coverage per sample ranging from 3,650,980 for the lowest quantile and 31,137,429 for the highest quantile. Data was normalized with a variance-stabilizing transformation (DESeq2 version 1.10.1) 31 prior to Principal Component Analysis (PCA) in R (version 3.2.4 and Biobase version 2.30.0 and 2.42.0 ${ }^{32}$ ). PCA was carried out using the R method (prcomp). Differential expression was calculated using DESeq2 (version 1.10.1) ${ }^{31}$. The results from DESeq2 can be found in Supplementary Tables 2 and 4. Heatmaps were generated in R using normalized read counts (variance-stabilizing transformation). 
Hermaphrodites that have interacted with males receive male sperm as a result of mating and male-sperm derived transcripts were detected in our data (Extended Data Fig. 1). To focus on the effect of sexual interactions of the hermaphrodites, we developed a list of male-enriched genes that were excluded from future analysis. Briefly, we used DEseq2 to calculate differential expression between the WT males and the $g l p-1(e 2144)$ hermaphrodites that never experienced a sexual interaction (day 3 and 7 of life were combined for this analysis). Genes that were expressed more highly in WT males ( $\log _{2}($ fold change $)>0$, adjusted $p$-value $\left.\leq 0.05\right)$ were excluded from the datasets. 5,355 genes met this threshold and were called as male-enriched. This filtered dataset was then carried through our standard RNA-seq pipeline (see above). Both the non-filtered datasets and the datasets in which the male-enriched genes were removed from the analysis are available as supplemental data sets and their analyses are included in Supplementary Table 2 and 4.

All code is publicly available online (https://github.com/brunetlab/Booth-et-al.-2020).

\section{Gene set enrichment}

WormCat ${ }^{33}$ was used to determine the gene set enrichment for the RNA-seq and microarray results. Genes were identified as significant by SAM (see below) and DESeq2 (see above). For the RNA-seq, genes were called as differentially expressed if the adjusted $p$-value was less than 0.05. Significantly up- and down-regulated genes were input to WormCat.org using default settings. For gene sets with a very small number of genes (those up- and down-regulated on male-conditioned plates), we did not observe significant enrichment using the multiplehypothesis corrected $p$-values and instead present the Fisher's Exact Test for these gene sets. Complete lists of all WormCat gene set enrichment results, including both the Fisher's Exact Test and Bonferroni corrected $p$-values are presented in Supplementary Tables 3, 5, and 9.

\section{Lifespan assays}

C. elegans hermaphrodites used for lifespan assays were age-synchronized with a short (3-4 hour) egg-lay using young (day 3-5 of life), well-fed, adult parents. All worms were grown on NGM plates with streptomycin $(50 \mu \mathrm{g} / \mathrm{mL})$ and seeded with OP50-1 bacteria unless RNAi knockdown was performed (see below). 
For each assay, worms were scored as dead or alive and transferred to new plates daily during the reproductive period and then every other day. Worms were scored as dead if they did not respond to gentle, repeated prodding with a wire pick $(90 \% \mathrm{Pt}, 10 \% \mathrm{Ir})$ along different points of their body. Worms were scored as censored if they crawled off the media or died due to bagging (internal hatching) or vulval rupture. Data from these censored worms were included up until the point of censorship (see Supplementary Table 1 for all data).

For conditions in which the effect of sexual interactions was assessed, we used one of three methods, as indicated. For the long-term exposure method (described previously ${ }^{1,2}$ ), young males (day 1 to 2 of adulthood) were added to the hermaphrodites at the onset of adulthood. For lifespan experiments in which the hermaphrodites were exposed to males for their entire adulthood ${ }^{1}$, males were added in a 1:1 ratio with hermaphrodites and the number of males remained fixed, even as hermaphrodites began to die or censored. Male worms were replaced every other day at the time the hermaphrodites were transferred to new plates. Male stocks were set up approximately every other day for the entirety of the lifespan assay. For the lifespan experiments in which hermaphrodites were exposed to males for only one day ${ }^{2}$, young males were added in a 2:1 male:hermaphrodite ratio. Following 24 hours of exposure, hermaphrodites were moved to new plates and did not encounter a male again throughout their lifespan. For the male-conditioned media lifespan assays, hermaphrodites were transferred on to male-conditioned plates (MCP) from late L4 stage and stayed on MCP for the remainder of their lives. Maleconditioned plates were prepared throughout the course of the lifespan assays: 30 Day 1 of life males (fog-2(q71), essentially WT) were transferred onto each plate (35mm NGM plates). Two days later, the males were removed and hermaphrodites for lifespan assays were immediately transferred onto these male-conditioned plates.

Synchronized worms (hermaphrodites, feminized individuals etc.) were randomly assigned to the "no males" or "+ males" conditions by picking them onto fresh plates in an alternating manner to avoid selection bias. Similarly, the males used for mating with individuals of different genotypes or RNAi treatments were from the same sets of males in each assay and were allocated randomly in an alternating manner. For each single biological replicate, approximately 35 individuals were placed on each of 3-6 plates (each plate represents a technical replicate). The number of individuals per plate and number of technical replicates were chosen based on field standards ${ }^{34}$. 
For sterile mutants, slight modifications were made to the methods. The sterile $g l p-1(e 2144)$ mutant and WT control parents were used for an egg lay at the permissive temperature $\left(15^{\circ} \mathrm{C}\right)$. Following the egg lay, the individuals used for the assay were kept at $25^{\circ} \mathrm{C}$ for the remainder of the assay.

Lifespan data were plotted as Kaplan-Meier survival curves in Prism 8 and statistical analyses performed using the logrank (Mantel-Cox) test. The number of animals (n) used for each assay and the number of independent biological replicates $(\mathrm{N})$ can be found in Supplementary Table 1.

\section{RNAi knock-down}

To knock-down expression of specific genes, we fed worms HT115 (E. coli) bacteria expressing double strand RNA targeting a specific gene. Worms were cultured on NGM containing ampicillin $(100 \mu \mathrm{g} / \mathrm{mL}$, Sigma) and IPTG $(0.4 \mathrm{mM}$, Invitrogen $)$. During development, worms were fed HT115 bacteria (grown to stationary phase, RNAi expression induced for 2-4 hours with $0.4 \mathrm{mM}$ IPTG, and the bacteria concentrated to $20 \mathrm{x}$ ) carrying control empty vector (EV). Upon adulthood (day 3 of life), worms were placed onto plates with HT115 bacteria (grown to stationary phase, RNAi expression induced for 2-4 hours with $0.4 \mathrm{mM}$ IPTG, and the bacteria concentrated to 20x) carrying the appropriate RNAi clone. RNAi clones in HT115 E. coli were isolated from the Ahringer RNAi library ${ }^{35}$ (a gift from A. Fire) or, if unavailable, from the Vidal RNAi library ${ }^{36}$ (Dharmacon). The inserts of the plasmids encoding the RNAi clones used in this study were sequenced to verify their identity. For all lifespan assays, the identity of the RNAi clone was blinded until the lifespan assay was completed.

We note that the RNAi construct that targets delm-2 shares high sequence similarity to two delm-2 paralogs - delm-1 and acd-1 (Extended Data Fig. 4). Interestingly, acd-1 expression was also induced by males (Fig. 1g, 2b and Extended Data Fig. 3d) and loss of acd-1 by RNAi knock-down partially protected hermaphrodites from male-induced demise (Fig. 4c, Extended Data Fig. 4b, and Supplementary Table 1). However, single mutations in these genes are not sufficient to protect hermaphrodites from male-induced demise (Extended Data Fig. 4b-f), suggesting that these channel genes may act redundantly or that the RNAi knock-down results in a greater loss of function either of a single gene or a combination of delm-2, delm-1, and acd-1. For simplicity, in the manuscript, we refer to this manipulation as delm-2 knock-down. 
To perform double RNAi, we combined equal amounts of bacteria expressing delm-2 targeting dsRNA and $a c b p-3$ targeting dsRNA. This was compared to control RNAi bacteria (with empty vector) and to single RNAi knock-down. The single RNAi knock-down for these experiments were diluted 50\% using control (empty vector) RNAi expressing bacteria. We note that RNAi knock-down does not result in complete loss of function (i.e. it is not a null). Therefore, a caveat to the interpretation of double RNAi results is that effects on lifespan may be due to intensifying the loss of function in a single pathway rather than targeting two parallel pathways.

\section{RNAi-based screen}

To determine whether the male-induced gene expression changes in hermaphrodites functionally contribute to their premature demise, we performed a targeted RNAi-based screen. The specific genes that we included in our screen were chosen because they were up-regulated in multiple datasets: $g l p-1$ sterile hermaphrodites for long and short interactions (Fig. 1g) and WT or feminized individuals mated with males for two hours ${ }^{13}$ (Extended Data Fig. 1d). We also included several genes that were highly male-enriched (lys-3, C29F7.2, T02B5.3, and T16G1.6) and that were not significantly enriched $(\mathrm{kgb}-1$ and $\mathrm{K09C4.5)}$ as controls to test whether genes that we filtered from our RNA-seq analysis (see above) were functionally important for maleinduced demise. None of these genes significantly extended hermaphrodite lifespan when knocked-down (Supplementary Table 1). As a positive control, we used RNAi knock-down of the male-induced demise gene $u t x-1^{1}$. RNAi treatments were blinded until the last animals died.

For the screen, we performed lifespan assays using a single plate of approximately $35 \mathrm{~N} 2$ (wild-type) hermaphrodites for each "no males" condition and two plates of approximately 18 N2 (wild-type) hermaphrodites and 18 him-5(e1467) males for each "+ males" condition. For details on the lifespan assays, see above.

\section{Microarrays}

glp-1(e2141) hermaphrodites were mated with wild-type or fer-6(hc6) males starting on day 1 of adulthood for 24 hours in a 2:1 male to hermaphrodite ratio. fer-6(hc6) males do not transfer sperm but have normal seminal fluid and copulation during mating ${ }^{3,17} .200$ hermaphrodites were collected on day $2 / 3$ of adulthood. Four biological replicates of wild-type males mated $g l p-1$ 
hermaphrodites were collected on day 2 of adulthood, another three replicates were collected on day 3 of adulthood. All six replicates of fer- 6 male mated $g l p-1$ hermaphrodites were collected on day 3 of adulthood.

Because male pheromone-induced demise depends on an intact germline ${ }^{11}$, male pheromoneinduced expression changes might be missed in our RNA-seq and microarray transcriptomic measurements using glp-1 (germline-less) animals. Therefore, we identified male pheromoneinduced expression changes using WT N2 hermaphrodites. Synchronized late L4 N2 hermaphrodites were picked on to $35 \mathrm{~mm}$ plates (control and male-conditioned plates [conditioned by 30 young fog-2 males for two days, see above]). 30 worms per plate—about 180 worms in total-were used for each biological replicate. Hermaphrodites were transferred on to freshly seeded or male-conditioned plates every two days, and collected on day six for RNA extraction. Four biological replicates were performed. Interestingly, male-conditioned plates induced hermaphrodite gene expression changes that were very similar to the transcriptional response of males to male-conditioned plates (up-regulated genes $p$-value $=0.00057$ and downregulated genes $p$-value $=4.96 \times 10^{-5}$, hypergeometric test $)^{11}$.

RNA was extracted by the heat vortexing method. Two-color Agilent microarrays were used for expression analysis. Significantly differentially expressed gene set were identified using $\mathrm{SAM}^{37}$. Additional analysis was performed in $\mathrm{R}$ and all $\mathrm{R}$ code is publicly available online (https://github.com/brunetlab/Booth-et-al.-2020).

Microarray data can be found in Supplementary Tables 6-8 and PUMAdb (http://puma.princeton.edu):

glp-1 hermaphrodites mated with wild-type males:

https://puma.princeton.edu/cgi-bin/exptsets/review.pl?exptset_no=7345

glp-1 hermaphrodites mated with fer-6 males:

https://puma.princeton.edu/cgi-bin/exptsets/review.pl?exptset_no=7346

N2 hermaphrodites on male-conditioned plates:

https://puma.princeton.edu/cgi-bin/exptsets/review.pl?exptset_no=7351

\section{Comparison of gene expression results}

The transcriptomic experiments (Fig. $1 \& 2$ ) were performed by different researchers using different experimental setups (e.g. the ratio of males to hermaphrodites) and nematode strains. 
To compare the RNA-seq and microarray gene expression results, we: calculated Pearson's correlations of the fold changes for the genes measured in the different experiments (Extended Data Fig. 3a), identified the genes that are common between the microarray and RNA-seq results and performed hypergeometric tests (Extended Data Fig. 3b), and compared gene set enrichments (Extended Data Fig. 3c). These comparisons revealed that the RNA-seq and microarray yielded highly similar results at the whole-genome and specific gene levels, highlighting the robustness of the male-induced demise phenomenon and our data.

\section{Alignment of delm-2 RNAi targeting sequence}

The delm-1 and acd-1 unspliced transcript sequences were downloaded from WormBase (WS275) and aligned to the Ahringer library RNAi construct that targets delm-2 using Clustal $\mathrm{Omega}^{38}$. The pairwise alignments were visualized using JalView ${ }^{39}$.

\section{Transcription factor binding enrichment}

ChIP-Atlas ${ }^{40}$ was used to determine the enrichment of ChIP-seq peaks at the male sperm, seminal fluid, and pheromone regulated genes. These differentially expressed genes were identified by microarrays (see above). The ChIP-seq peak significance threshold was set to 100 (equivalent to a Q-value $<1 \times 10^{-10}$ ) and ChIP-seq peaks from the groups "TFs and others" and "All cell types" were used. ChIP-seq peaks within $2 \mathrm{~kb}^{41}$ up- and downstream of the transcription start site were considered for each differentially expressed gene. The presence and strength of transcription factor peaks for select genes (Fig. 3d) was also determined using ChIP-Atlas on default settings. We note that these ChIP-seq experiments were performed in whole worms at various developmental stages and under different conditions. Thus, it is important to keep in mind that these transcription factor-gene regulatory connections may not represent the situation in a given adult tissue undergoing male-induced demise. The complete list of enriched transcription factor binding is in Supplementary Table 10.

\section{Motif analysis}

To calculate known and de novo motif enrichment within the promoter regions of differentially expressed genes, we used the Homer" function "findMotifs.pl" with parameters “- 
start -300 -end 300" (we also tested a larger region with "-start -2000 -end 2000" and found similar results).

To identify whether specific motifs were enriched in the promoter regions of differentially expressed genes, we first downloaded the PSWM files for the FOS, PBX3, and PQM-1 binding motifs from Homer ${ }^{42}$. We used the ChIPSeeker ${ }^{43}$ function "getPromoters" with parameters "TxDb=TxDb.Celegans.UCSC.ce11.refGene, upstream=300, downstream=300" to assign promoters to genes from lists of differentially expressed genes generated from the RNA-seq data with a statistical significance threshold of FDR $<0.05$ (we also tested a larger region with "upstream $=2000$, downstream=500" and found similar results). The resulting bed files were converted to FASTA format with bedtools "getfasta" 44 to be made compatible with the MEME suite AME (McLeay and Bailey, 2010). The MEME Suite AME ${ }^{45}$ (with default parameters "Average odds score" and "Fisher's exact test" using shuffled input sequences as the control) was used with to determine statistical enrichment of the chosen motifs. We found that the PQM1 motif was significantly enriched at the male sperm upregulated $\left(p=3.87 \times 10^{-22}\right)$ and downregulated $\left(p=1.23 \times 10^{-20}\right)$ genes, the male seminal fluid upregulated $\left(p=5.87 \times 10^{-3}\right)$ and downregulated $\left(p=9.8 \times 10^{-19}\right)$ genes, and the male pheromone upregulated genes $(p=4.93 \times$ $\left.10^{-6}\right)$. 
bioRxiv preprint doi: https://doi.org/10.1101/2020.06.30.181008; this version posted July 1, 2020. The copyright holder for this preprint (which was not certified by peer review) is the author/funder, who has granted bioRxiv a license to display the preprint in perpetuity. It is made

\section{Figure 1} available under aCC-BY-NC-ND 4.0 International license.

a

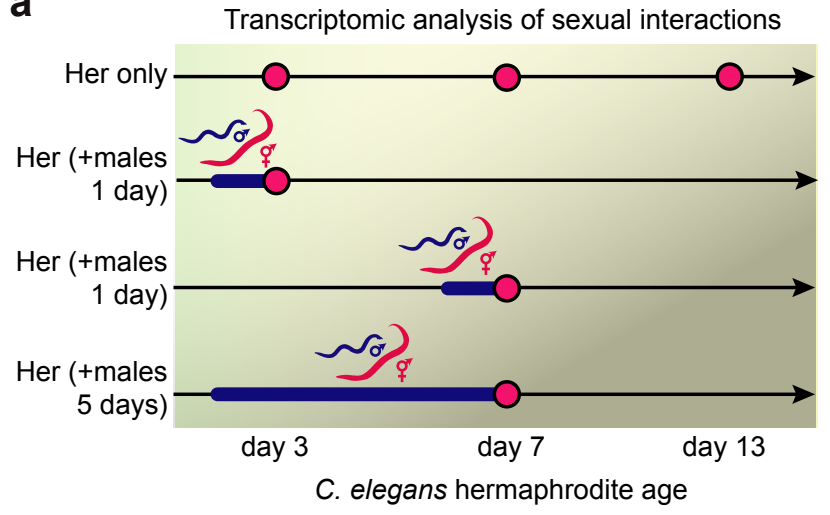

b

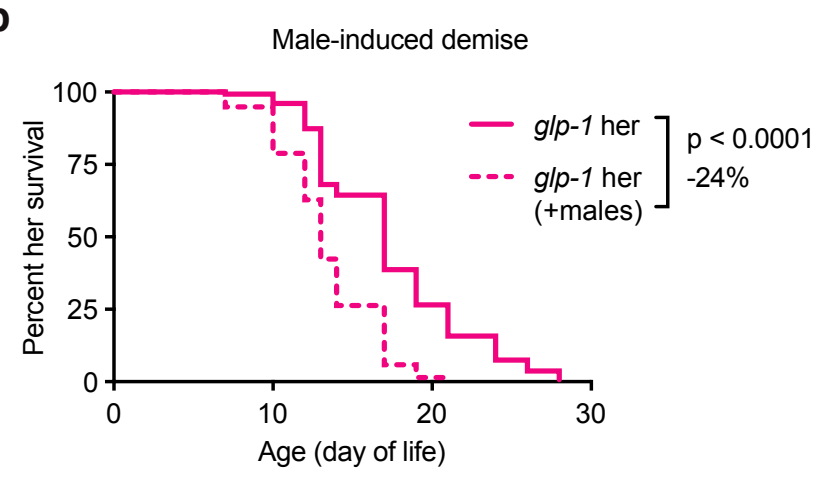

C

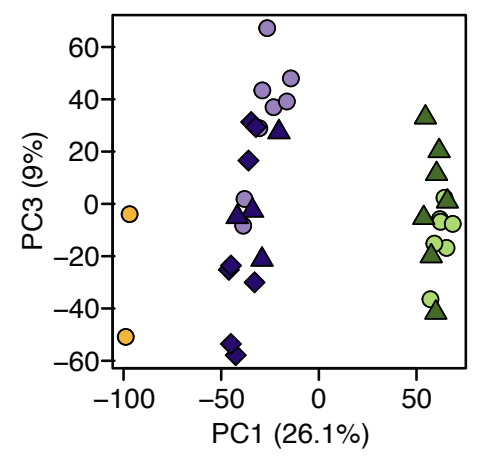

f

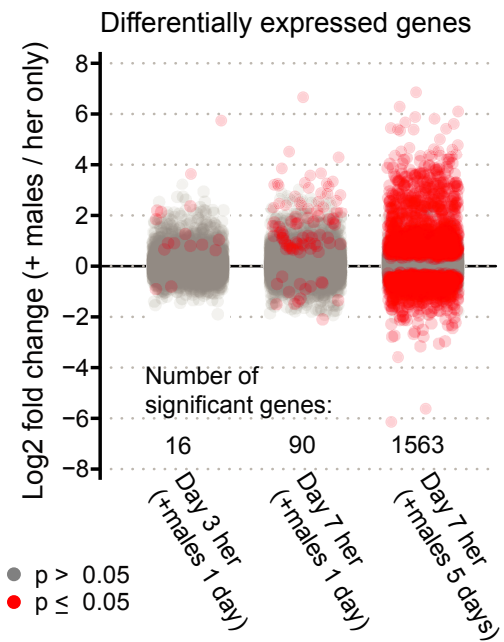

d

O Day 3

- Day 7

O Day 13

O Her only

$\triangle$ Her (+males 1 day)

$\diamond$ Her (+males

5 days)

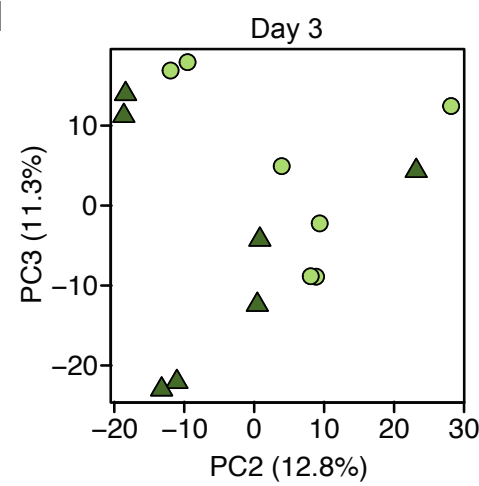

g Common differentially expressed genes

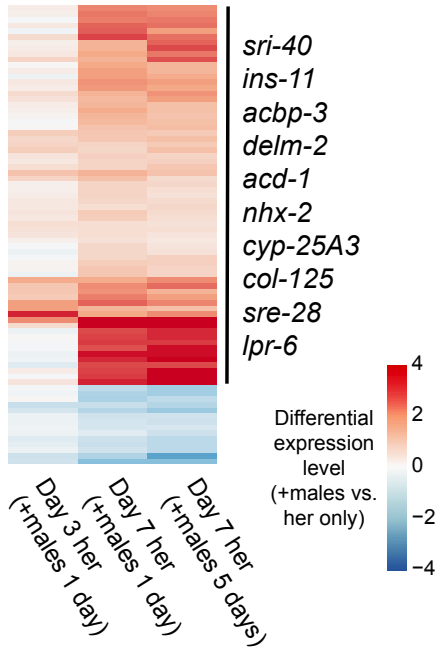

h

Gene category enrichment: common differentially expressed genes

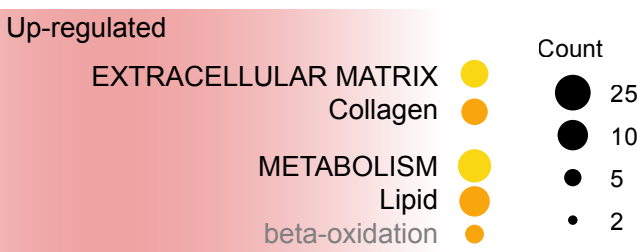

TRANSMEMBRANE TRANSPORT p-value Lipid $\quad 10-10$

STRESS RESPONSE 0.001

0.05 


\section{Figure 1: Sexual interactions induce premature death and specific transcriptional changes}

\section{in C. elegans hermaphrodites}

(a) Scheme describing the ages of the glp-1(e2144) hermaphrodites (Her) used in the RNA-seq experiments and the length of sexual interactions with males. The ages (day of life) of the hermaphrodites at the time of sample collection are indicated with a pink dot and the period of time (one day vs. five days) with males is indicated by a blue line. (b) The presence of males starting at adulthood shortens the lifespan of sterile $g l p-1$ (e2144) C. elegans hermaphrodites ( $p<$ $0.0001,-24 \%$ change in median lifespan). Lifespan data are plotted as Kaplan-Meier survival curves, the $p$-value was calculated using Mantel-Cox log-ranking, and 79-154 hermaphrodites were tested. A complete list of all lifespan assay results is in Supplementary Table 1. (c-e) Principal Component Analysis (PCA) of the normalized read counts from the hermaphrodite transcriptomes after removal of the male-enriched genes (see Materials and Methods and Extended Data Fig. 1a, b). In panel c, the data from all hermaphrodite age groups are used and in panels $\mathrm{d}$ and e the read counts from only day three or day seven were normalized and analyzed. (f) The male-induced hermaphrodite gene expression changes following one day or five days of interaction between the sexes. After filtering the data for male-enriched genes, the $\log _{2}$ (fold change) for all detected genes is displayed and genes that are significantly differentially expressed $(p \leq 0.05)$ in the presence of males are shown in red. Complete differentially expression analysis results are in Supplementary Table 2. (g) A heatmap of the genes that are significantly differentially expressed in hermaphrodites in response to the presence of males for both one day (day 3 or 7 hermaphrodites) and five days. The data are displayed as $\log _{2}$ (fold change). (h) Selected, enriched gene categories from the differentially expressed genes that are common between hermaphrodites that interact with males for one and five days. Gene annotations are nested with the broadest categories listed in all capital letters and the middle categories listed with the first letter capitalized, and the most specific categories in grey. Complete gene set enrichment analysis results are in Supplementary Table 3. (i) The results of our RNAi-based screen for functionally important hermaphrodite genes in maleinduced demise. The colors of the circles indicate the change in maximal lifespan relative to control, empty vector RNAi. The sizes of the circles indicate the p-values calculated using MantelCox log ranking. Genes are ordered from smallest to largest change in maximal lifespan in the presence of males, using $p$-value to break ties. The screen hits are indicated with red gene name labels. A complete list of all lifespan data is in Supplemental Table 1. 
bioRxiv preprint doi: https://doi.org/10.1101/2020.06.30.181008; this version posted July 1, 2020. The copyright holder for this preprint (which was not certified by peer review) is the author/funder, who has granted bioRxiv a license to display the preprint in perpetuity. It is made

\section{Figure 2}

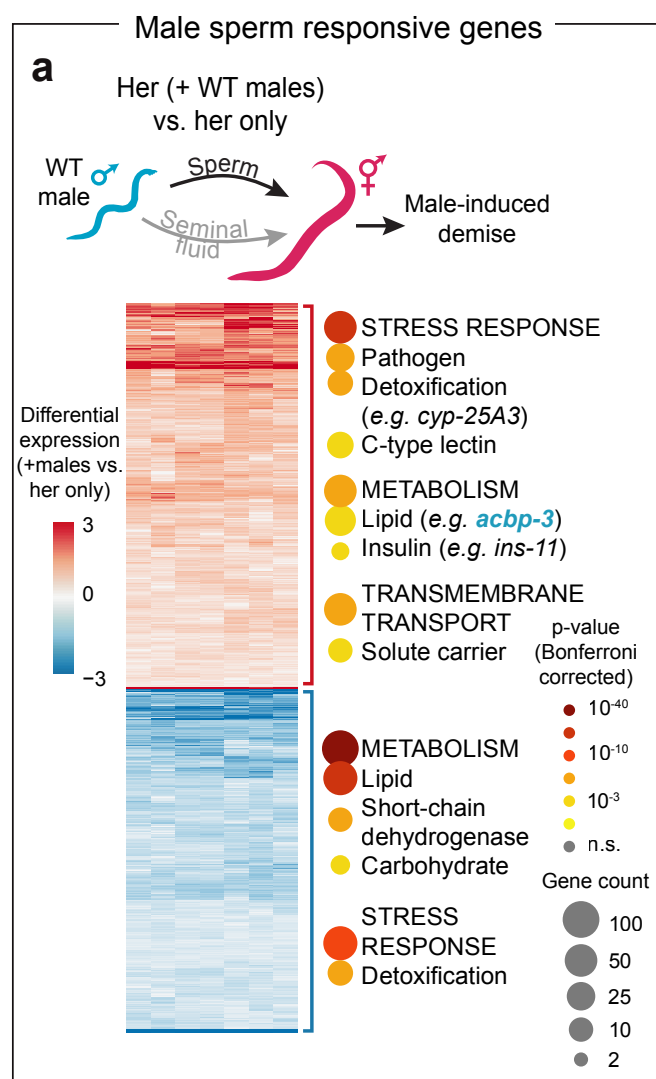

d

acbp-3: acyl-CoA binding protein

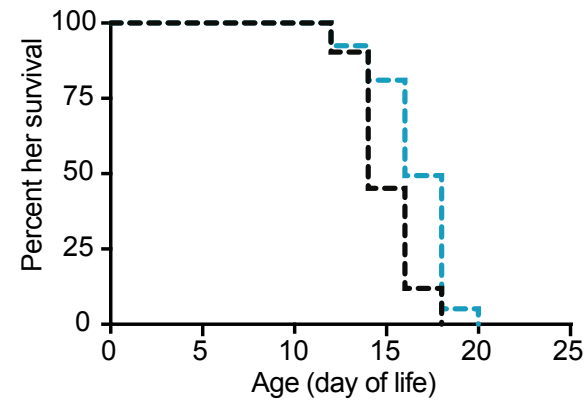

-- ctrl. RNAi (+males) $] p<0.0001$ - - acbp-3 RNAi (+males) $]+14 \%$

g

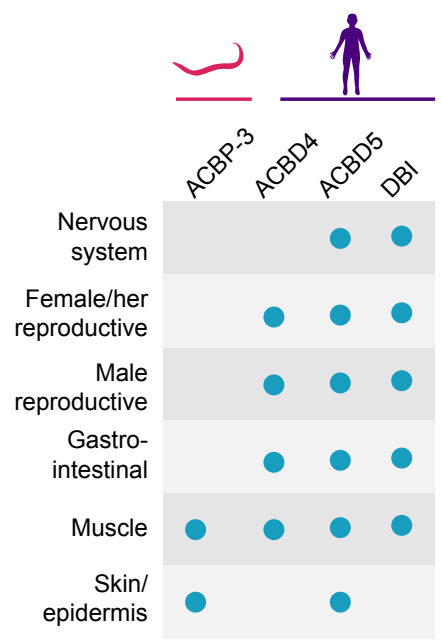

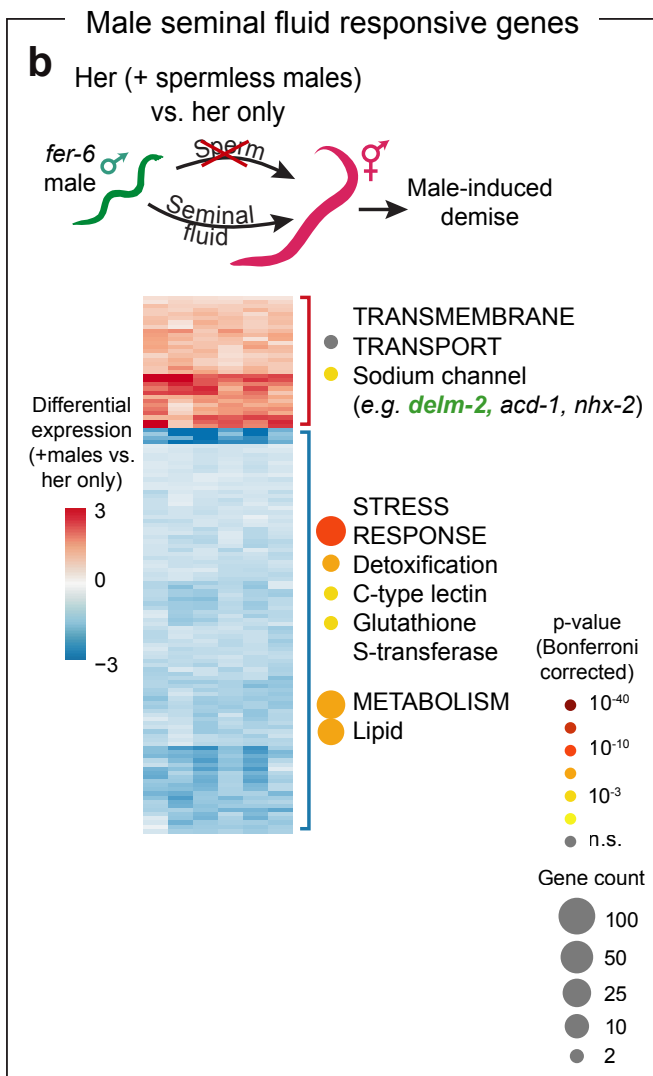

e

delm-2: degenerin-like sodium channel

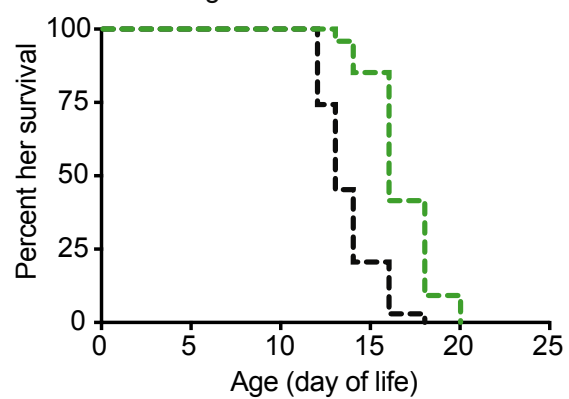

--- ctrl. RNAi (+males) $7 p<0.0001$ -. delm-2 RNAi (+males) $+23 \%$

h

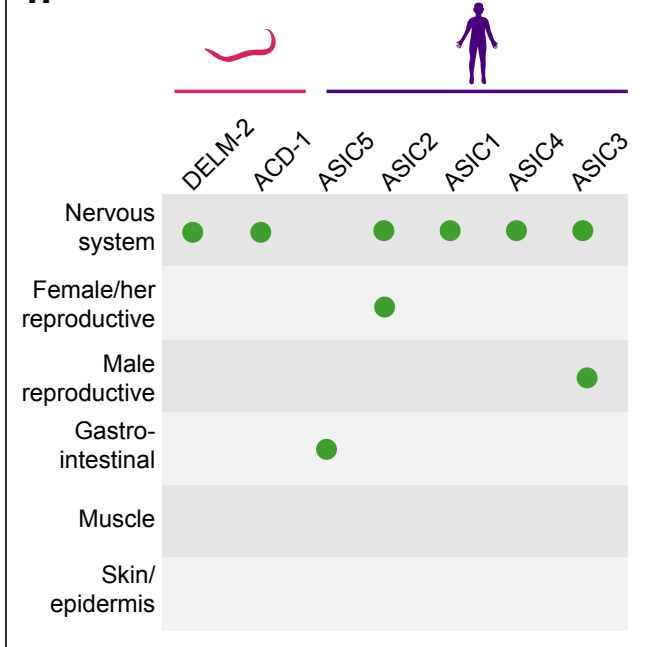

Male pheromone responsive genes

C Male-conditioned plates vs. normal plates
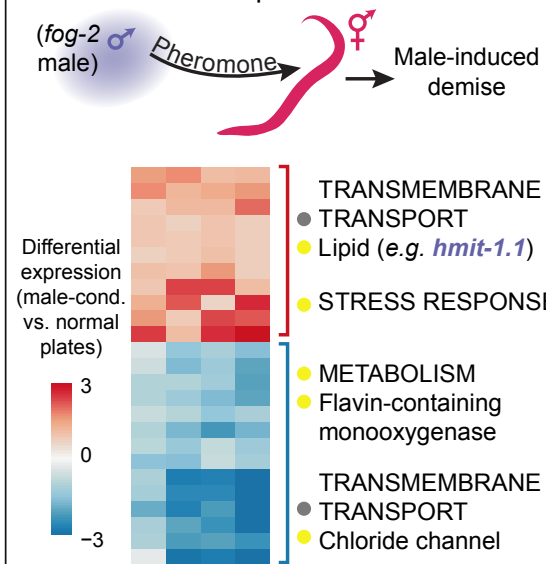

p-value
(Fisher's
exact)

- $10^{-40}$

$-10^{-10}$

(

- n.s.

Gene count

100

50

25

f

hmit-1.1: solute carrier/transporter

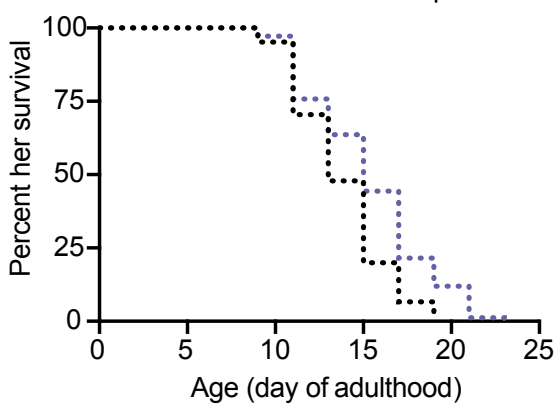

.... ctrl. RNAi + male-cond. plates $\quad 7 p=0.0003$ .... hmit-1.1 RNAi + male-cond. plates $]+15 \%$

i

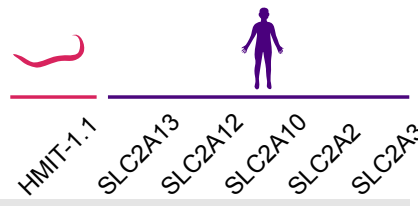

Nervous

system

Female/her reproductive

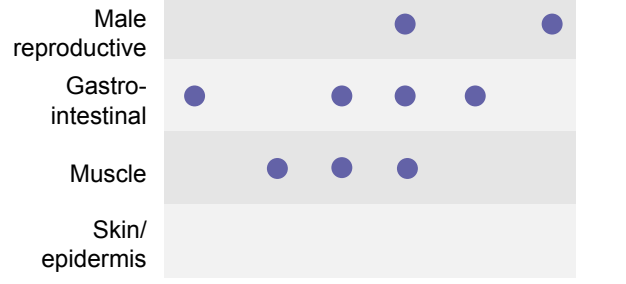




\section{Figure 2: Identification of male sperm, seminal fluid, and pheromone induced genes}

(a-c) Heatmaps and gene set enrichment of the microarray results. Heatmap data are displayed as $\log _{2}$ (fold change). Selected, enriched gene categories for the upregulated (red bracket) and downregulated (blue bracket) genes are shown to the right of each heatmap. Gene category annotations are nested with the broadest categories listed in all capital letters and categories within the broadest categories listed below, with the first letter capitalized. The number of differentially expressed genes in indicated by the size of the circles and enrichment significance by color. The complete results from the microarrays and the gene set enrichment can be found in Supplementary Tables 6 to 9. (a) The male sperm-regulated hermaphrodite glp-1[e2141] genes that were identified by microarray as significantly changing in response to mating with wild-type males (that transfer sperm and seminal fluid) but not changing expression in response to mating with males that do not make sperm (fer-6[hc6]). The WT male mated hermaphrodite microarray results are shown. (b) A heatmap of the putative male seminal fluid-regulated hermaphrodite genes that were identified by microarray as significantly changing both in response to mating with WT males that transfer sperm and seminal fluid and also with males that transfer seminal fluid but do not make sperm (fer6[hc6]). The gene expression results from fer-6 male mated hermaphrodites are shown. (c) A heatmap of the hermaphrodite genes that are significantly differentially expressed following exposure to male pheromones (from male-conditioned plates) compared to normal plates. Note that the $p$-values displayed in panel c are from the Fisher's Exact Test whereas those in panel a and $\mathrm{b}$ are the more stringent Bonferroni corrected $p$-values. (d) RNAi knock-down of the sperminduced gene $a c b p-3$ extends the lifespan of hermaphrodites in the presence of males (blue dashed line) compared to empty vector control (black dashed line) in the presence of males $(p<0.0001)$. (e) RNAi knock-down of the seminal fluid-induced gene delm-2 (green dashed line) extends the lifespan of hermaphrodites experiencing a sexual interaction with males $(p<0.0001)$. We note that the delm-2 targeting RNAi construct is highly similar to paralogs of delm-2-acd-1 and delm-1 (Extended Data Fig. 5 and 6). (f) RNAi knock-down of the male-conditioned plate induced gene hmit-1.1 (purple dashed line) extends the lifespan of hermaphrodites when they are cultured on male-conditioned plates $(p=0.0003)$. Lifespan data for panels d-f are displayed as Kaplan-Meier survival curves and $p$-values calculated using Mantel-Cox log-ranking. Percent change in median lifespan is shown for each lifespan compared to control. 107-124 hermaphrodites were used in each condition. A complete list of all lifespan assay results is in Supplemental Table 1. (g-i) The 
bioRxiv preprint doi: https://doi.org/10.1101/2020.06.30.181008; this version posted July 1, 2020. The copyright holder for this preprint (which was not certified by peer review) is the author/funder, who has granted bioRxiv a license to display the preprint in perpetuity. It is made available under aCC-BY-NC-ND 4.0 International license.

tissues that express the male-induced genes acbp-3 (panel g), delm-2 (panel h), and hmit-1.1 (panel i) and their human orthologs. The human orthologs shown are the top 5 BLASTp hits. Tissuespecific protein expression is indicated for each ortholog in C. elegans (www.wormbase.org, version WS275) and humans (www.proteinatlas.com ${ }^{46,47}$ ). 
bioRxiv preprint doi: https://doi.org/10.1101/2020.06.30181008; this version posted July 1, 2020. The copyright holder for this preprint (which was not certified by peer review) is the author/funder, who has granted bioRxiv a license to display the preprint in perpetuity. It is made available under aCC-BY-NC-ND 4.0 International license.

\section{Figure 3}

a

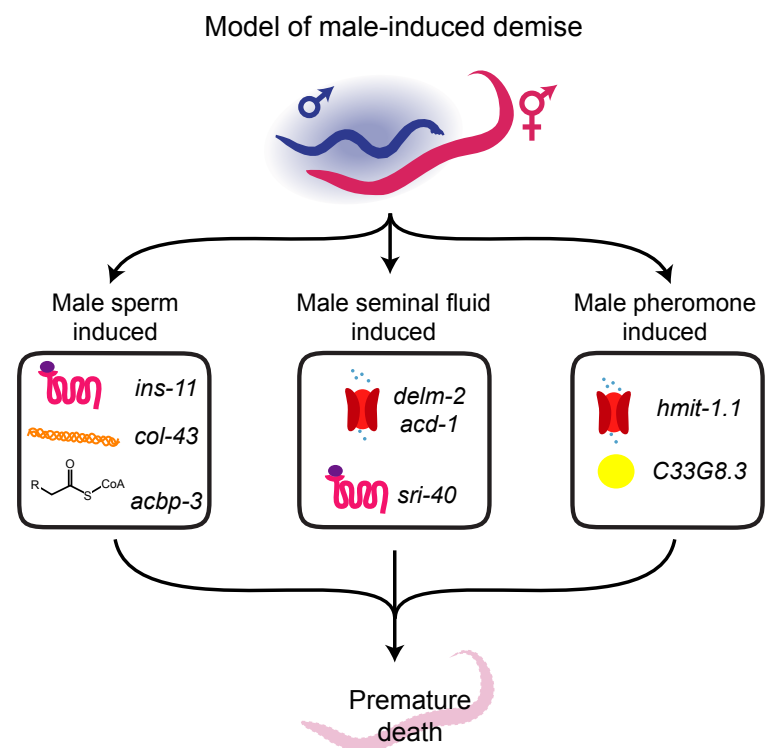

b

acbp-3 + delm-2

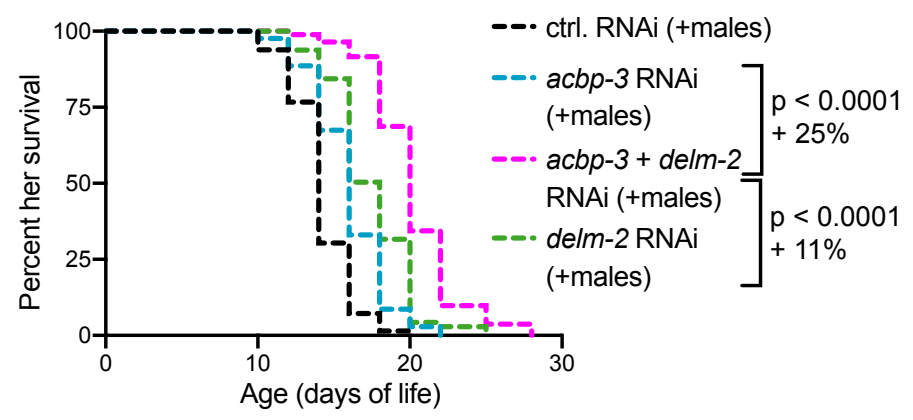

C

acbp-3 + delm-2
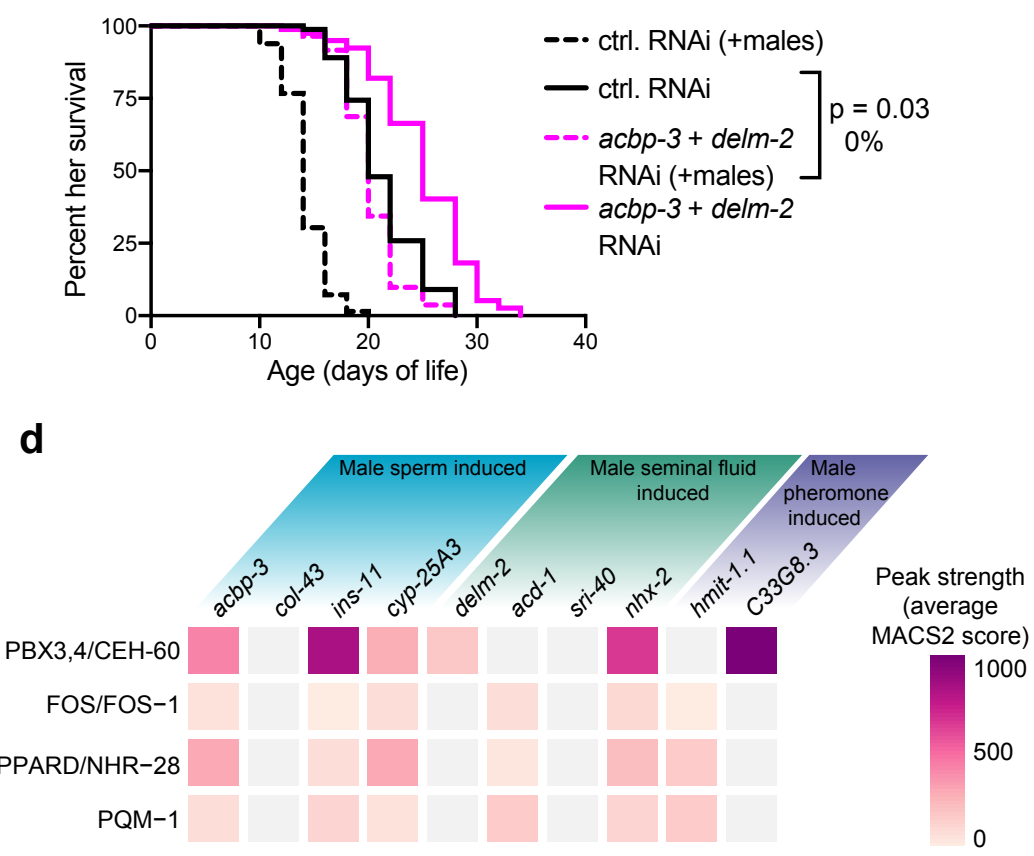


\section{Figure 3: Strategies to target multiple male-induced demise pathways}

(a) A summary of the transcriptomics and male-induced demise lifespan data showing that male sperm, seminal fluid, and pheromones each induce a different set of functionally important maleinduced demise gene in hermaphrodites. (b) Knock-down of $a c b p-3$ or delm-2 individually by RNAi partially protected hermaphrodites from male-induced demise (acbp-3: $p<0.0001,14 \%$ increase in median lifespan and delm-2: $p<0.0001,29 \%$ increase in median lifespan compared to control RNAi). Knock-down of $a c b p-3$ and delm-2 simultaneously by double RNAi (dashed pink line) protected hermaphrodites from male-induced demise to a greater extent than knock-down of either $a c b p-3$ or delm-2 alone ( $p<0.0001,25 \%$ and $11 \%$ increase in median lifespan, respectively). (c) Knock-down of $a c b p-3$ and delm-2 simultaneously extended hermaphrodite lifespan. In the presence of males (the dashed lines), loss of acbp-3 and delm-2 increased median lifespan by $43 \%$ $(p<0.0001)$. This resulted in a lifespan that was comparable to control hermaphrodites in a singlesex setting (black, solid line vs. dashed, pink line: $p=0.03$, no change in median lifespan). In the absence of males, loss of $a c b p-3$ and delm-2 increased median lifespan by $25 \%(p<0.0001)$ compared to control RNAi (solid, black line). Lifespan data are plotted as Kaplan-Meier survival curves, the $p$-value was calculated using Mantel-Cox log-ranking, and percent change in median lifespan is shown. 105-114 hermaphrodites were tested. A complete list of all lifespan data is in Supplementary Table 1. (d) The average MACS2 score of transcription factor ChIP-seq binding peaks within $5 \mathrm{~kb}+/$ - of the transcription start site of male-induced functional important genes. A complete list of the results of the transcription factor enrichment analysis can be found in Supplementary Table 10. 


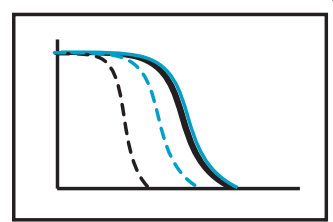

Specific

male-induced demise protection
Classes of protected or long-lived hermaphrodites:

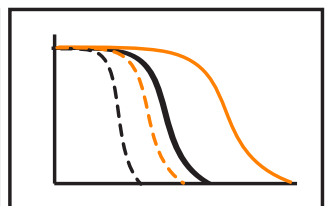

General

male-induced demise protection

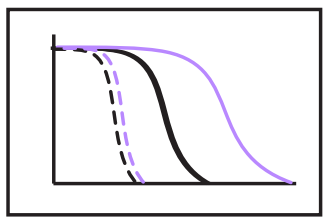

Male-induced demise

hypersensitive

$$
\begin{array}{ll}
\text { - Control her } & -- \text { Control her (+males) } \\
\equiv \text { Mutant or RNAi her } & \equiv \equiv \text { Mutant or RNAi her } \\
& \text { (+males) }
\end{array}
$$

C
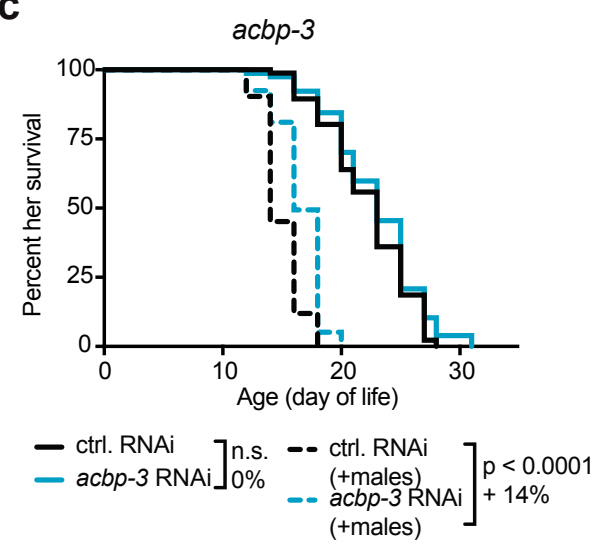

f

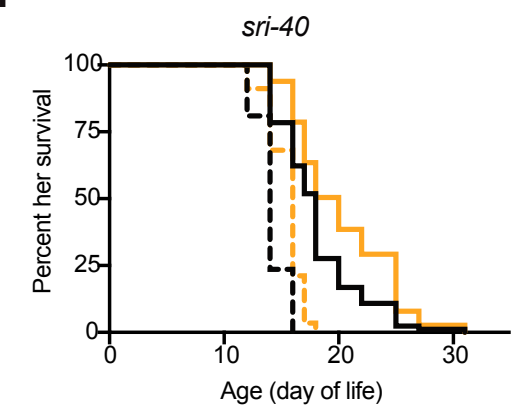

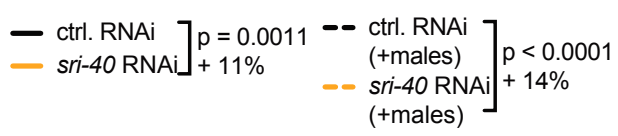

i
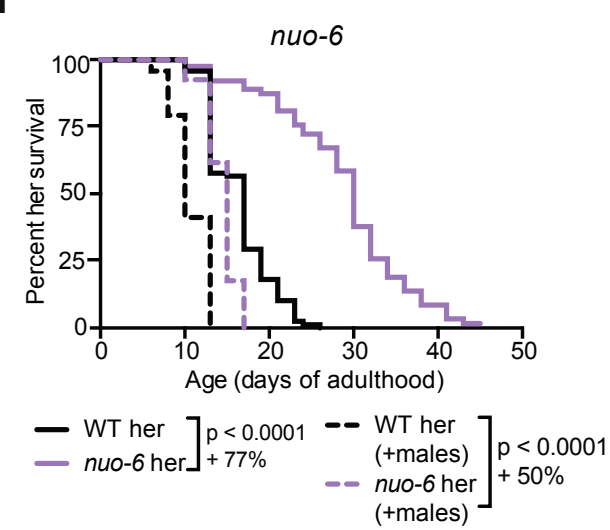

d

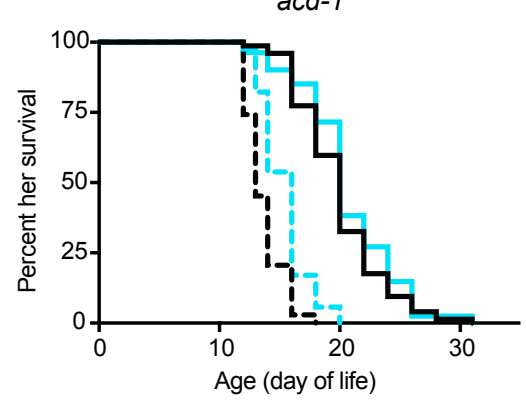

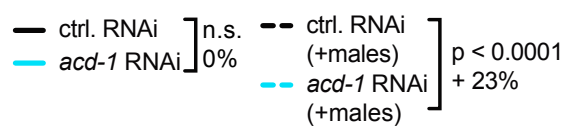

g

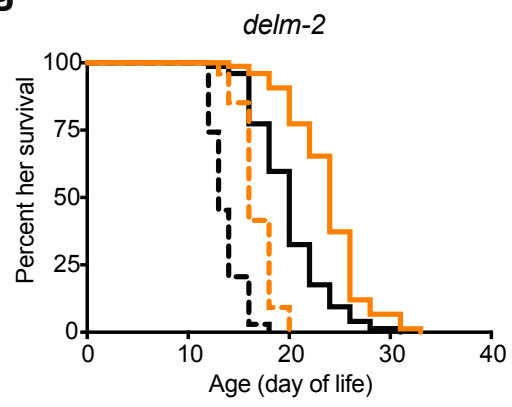

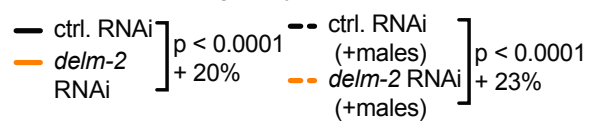

j

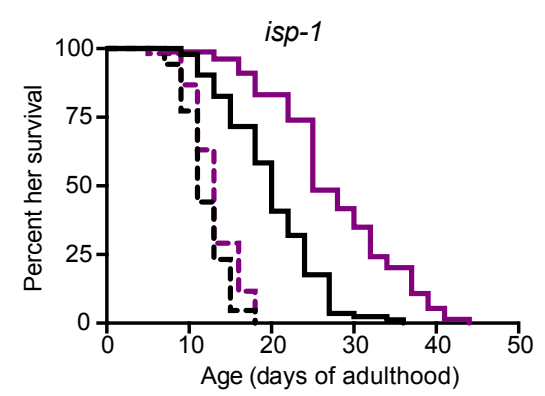

$\left.\left.\begin{array}{lr}\text { WT her } \\ \text { - isp-1 her }\end{array}\right] \begin{array}{lr}p<0.0001 \\ +25 \% & - \text { WT her } \\ & \text { (+males) } \\ & \text { isp-1 her } \\ (+ \text { males })\end{array}\right]+18 \%$ b

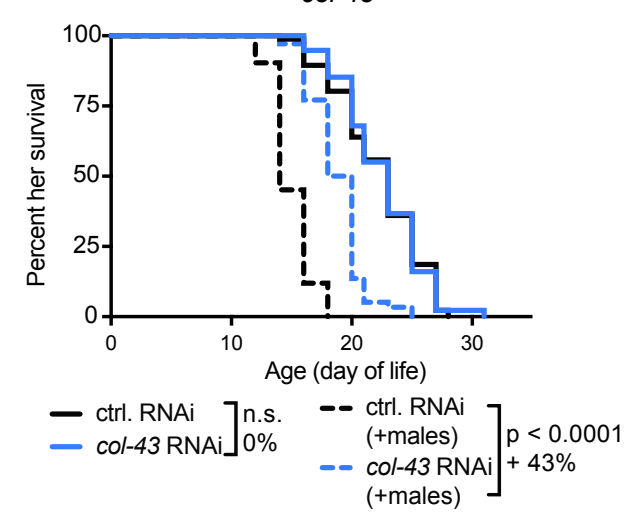

e

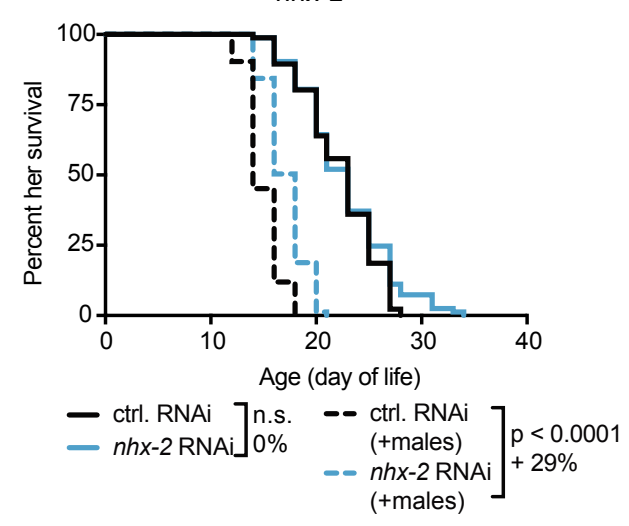

h

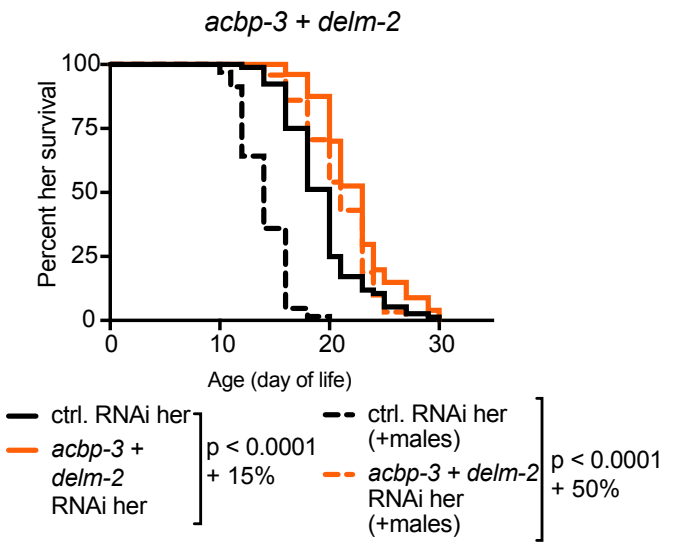

k

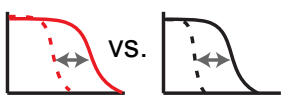

Effect of males relative to WT/control

WT her on acbp-3 \& delm-2 RNAi 0.52 Protected \begin{tabular}{ll} 
WT her on col-43 RNAi 0.37 \\
\hline
\end{tabular} acd-1 her 0.36

WT her on acd-1 RNAi 0.25

WT her on delm-2 RNAi 0.24

WT her on acbp-3 RNAi $\quad 0.09$

WT her on sri-40 RNAi 0.02

WT her on utx-1 RNAi -0.05

WT her on $n h x-2$ RNAi $\quad-0.12$

nuo-6(qm200) her -0.17

WT her on col-125 RNAi -0.36

daf-2(e1370) her -2.21 
Figure 4: Male-induced demise is mediated by specific genes, general health genes, and hijacking of conserved longevity pathways

(a) A scheme describing the different effects of lifespan extending knock-down and mutations in the presence and absence of males. (b-e) Knock-down of the collagen gene col-43 (b), the acylCoA binding protein gene $a c b p-3$ (c), the ion channel acd-1 (d), or the transporter $n h x-2$ (e) by RNAi partially protected hermaphrodites from male induced demise (dashed lines) but did not detectably extend lifespan in the absence of males (solid lines). We note that the male-induced demise lifespans in panel c (dashed lines) are also displayed in Fig. 2d and are displayed again here for comparison with hermaphrodite lifespan in the absence of males. (f-g) Knock-down of the serpentine receptor sri-40 (f) or the sodium channel delm-2 (g) extended hermaphrodite lifespan in both the presence (dashed lines) and absence (solid lines) of males. We note that the male-induced demise lifespans in panel $g$ (dashed lines) are also displayed in Fig. 2e and are displayed again here for comparison with the lifespans in the absence of males. (h) Knock-down of both delm-2 and acbp-3 simultaneously extended hermaphrodite lifespan in both the absence of males (solid lines) and in the presence of males (dashed lines). (i-j) Mutations in the mitochondria electron transport chain genes nuo-6(qm200) and isp-1(qm150) extended lifespan in a hermaphrodites only setting (solid lines). In the presence of males, nuо- 6 hermaphrodites lived longer than wild-type (panel e, dotted lines), but this extension was blunted compared to the extension of lifespan in the absence of males (see panel k). In the presence of males, isp-1 mutant hermaphrodites did not live longer than wild-type (panel j, dashed lines). All lifespan data are plotted as Kaplan-Meier survival curves, the $p$-values were calculated using Mantel-Cox log-ranking, and percent changes in median lifespan are shown for each comparison. 55-116 hermaphrodites were tested. A complete list of all lifespan data is in Supplementary Table 1. (k) A summary of the percent loss of lifespan in the presence of males in the mutant or RNAi knockdown compared to wild-type. The effects of males for each genetic manipulation relative to wildtype was calculated as 1 - (male-induced change in median lifespan of hermaphrodites with mutation or RNAi / male-induced change in median lifespan of control). Positive values indicate that the genetic manipulation resulted in greater protection from male-induced demise whereas negative values indicate hypersensitivity to male-induced demise. The daf-2(e1370) results were published previously ${ }^{1}$. All other lifespan results are from this manuscript (see Supplementary Table 1 and 11). 
bioRxiv preprint doi: https://doi.org/10.1101/2020.06.30.181008; this version posted July 1, 2020. The copyright holder for this preprint (which was not certified by peer review) is the author/funder, who has granted bioRxiv a license to display the preprint in perpetuity. It is made

\section{Extended Data Figure 1}

a

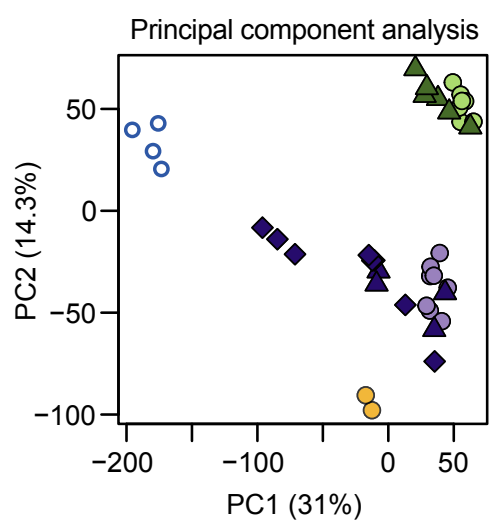

C

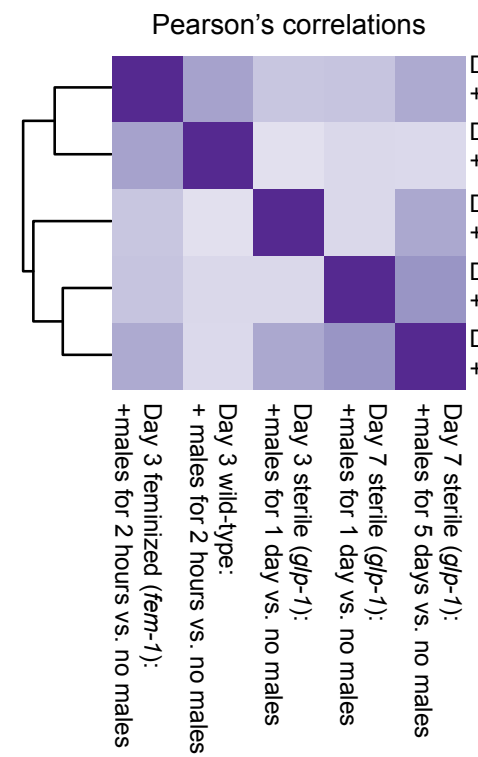

b

O Day 3

- Day 7

o Day 13

O Her only

$\diamond$ Her (+males

5 days)

$\triangle$ Her (+males

1 day)

o Males d

Day 3 feminized (fem-1):

+males for 2 hours vs. no males Day 3 wild-type:

+ males for 2 hours vs. no males

Day 3 sterile $(g / p-1)$ :

+males for 1 day vs. no males

Day 7 sterile $(g / p-1)$ :

+males for 1 day vs. no males

Day 7 sterile $(g / p-1)$ :

+males for 5 days vs. no males

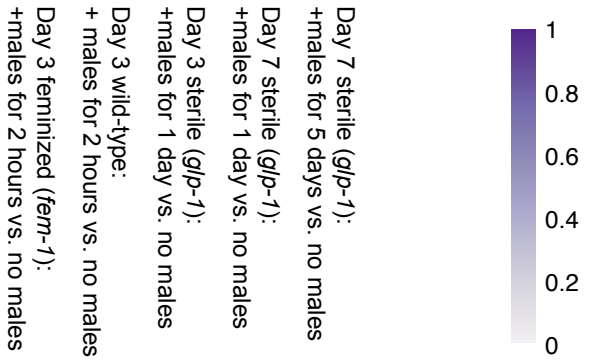

Common differentially expressed genes

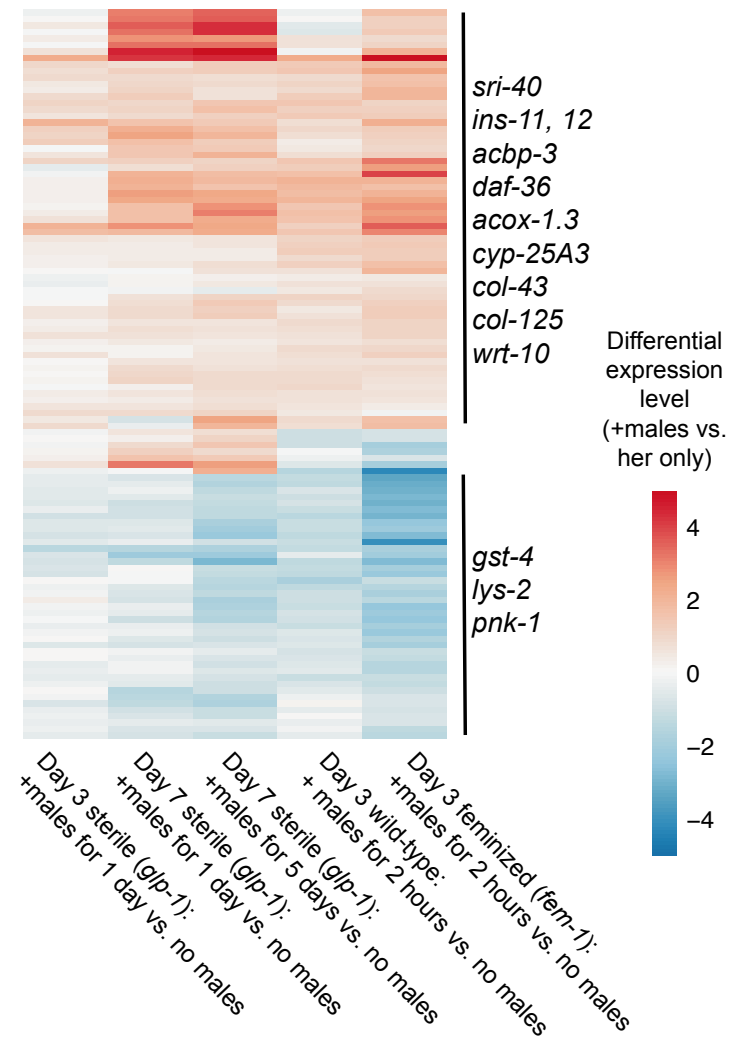

e

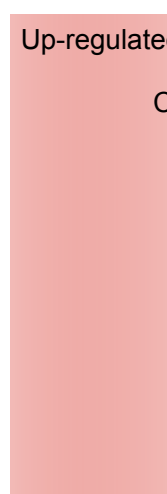

\section{CELL CYCLE}

Chromosome dynamics

meiotic functions

Meiotic/mitotic spindle

Other

DNA

Replication

Repair

CYTOSKELETON

Microtubule

tau tubulin kinase

SIGNALING

Phosphatase

other

$Y$ kinase

DEVELOPMENT

MAJOR SPERM PROTEIN

TRAFFICKING

ER/Golgi

METABOLISM

Mitochondria

ribosome

Lipid

beta-oxidation

STRESS RESPONSE

C-type lectin

Day 7 her
Male (+males 1 day)

vs. her vs. her only
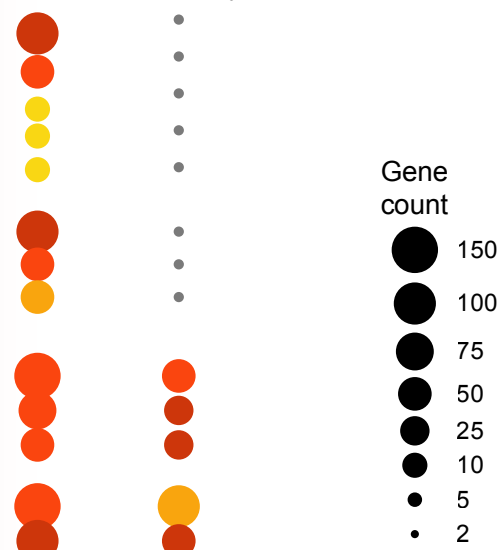

p-value

- $10-40$

- $10-20$

- $10-10$

- $10-5$

- 0.001

- 0.05

- n.s. 


\section{Extended Data Figure 1: RNA-seq of $C$. elegans hermaphrodites and males}

(a) Principal Component Analysis of the normalized read counts of the transcriptomes of $g l p$ 1(e2144) hermaphrodites and wild-type males prior to removal of the male-enriched genes (see Material and Methods). (b) Enriched gene categories in the genes expressed more highly in wildtype males versus $g l p-1$ hermaphrodites (left) and the genes expressed more highly in $g l p-1$ hermaphrodites that experienced a sexual interaction for one day (starting at day 6 of life) versus no sexual interaction (right). The number of differentially expressed genes in each gene set is indicated by the size of the circle and the significance of the enrichment by the color of the circle. Gene annotations are nested with the broadest categories listed in all capital letters and the middle categories listed with the first letter capitalized, and the most specific categories in grey. These two sets of differentially expressed genes share several enriched gene categories, including those linked with sperm such as the major sperm proteins, tau tubulin kinases ${ }^{33}$, and phosphatases/kinases ${ }^{48}$ suggesting that male sperm-derived transcripts are detected by wholeworm RNA-seq of mated hermaphrodites. (c) Pearson's correlation of the DEseq2 calculated $\log _{2}$ (fold change) differential expression of all shared, detected genes in wild-type hermaphrodites, sterile $g l p-1(e 2144)$ individuals, and feminized fem-1(hc17) individuals following a sexual interaction with males versus no sexual interaction. Data are from this manuscript and Booth et al. eLife 2019. (d) Heatmap of the genes that are differentially expressed in at least three of the five conditions. The data are displayed as $\log _{2}$ (fold change) and the complete results of the differential expression analysis can be found in Supplementary Table 2 and Booth et al. eLife 2019. (e) Enriched gene categories in the genes that are upregulated (red) or downregulated (blue) following sexual interactions with males in at least three of the five datasets from $g l p$ - 1 sterile hermaphrodites (this manuscript), fem-1 feminized individuals, and WT hermaphrodites ${ }^{13}$. Complete differential expression and gene set analysis results are in Supplementary Tables 2 to 5. 
a

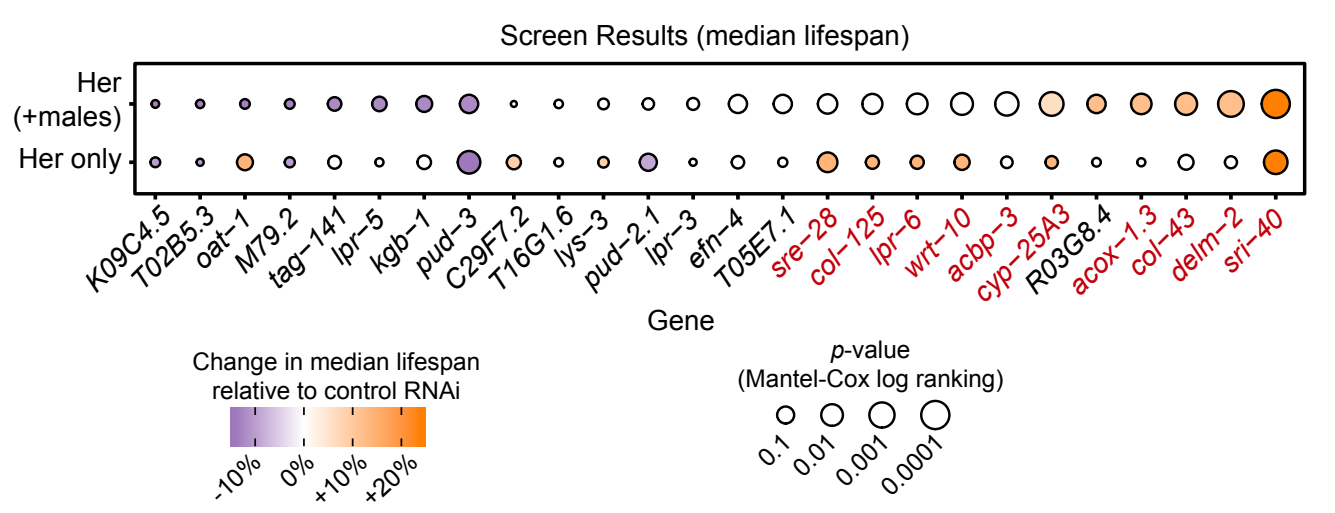

C

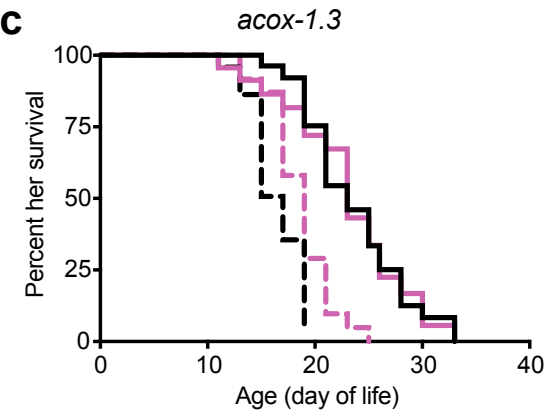

$\left.\begin{array}{rl}- \text { ctrl. RNAi } \\ - \text { acox-1.3 RNAi }\end{array}\right]$ n.s. $\left.\begin{array}{rl}-- & \text { ctrl. RNAi } \\ & (+ \text { males }) \\ -- & \text { acox-1.3 RNAi } \\ & (+ \text { males })\end{array}\right]+12 \%$

f

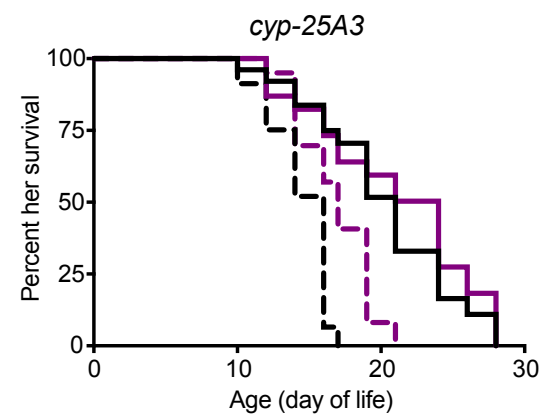

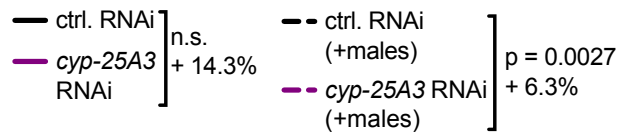

i

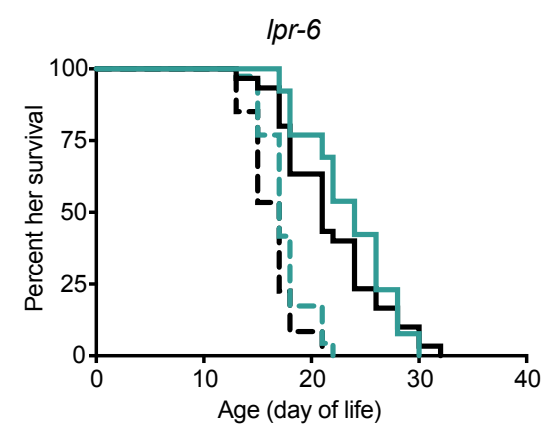

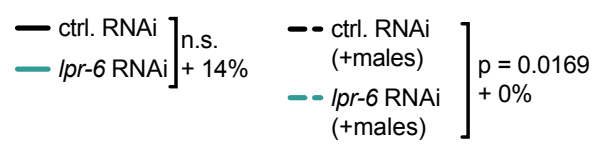

d

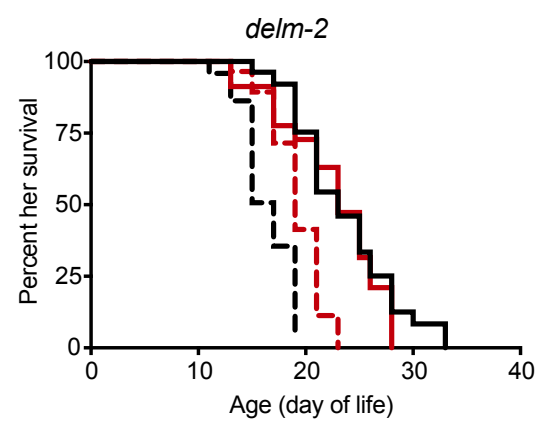

$\left.\begin{array}{cc}\text { - ctrl. RNAi } \\ \text { - delm-2 RNAi }\end{array}\right]$ n.s. $\begin{gathered}- \text { ctrl. RNAi } \\ \text { (+males) } \\ \left.-\begin{array}{c}\text { delm-2 RNAi } \\ \text { (+males })\end{array}\right] p=0.0007 \\ +12 \%\end{gathered}$

g

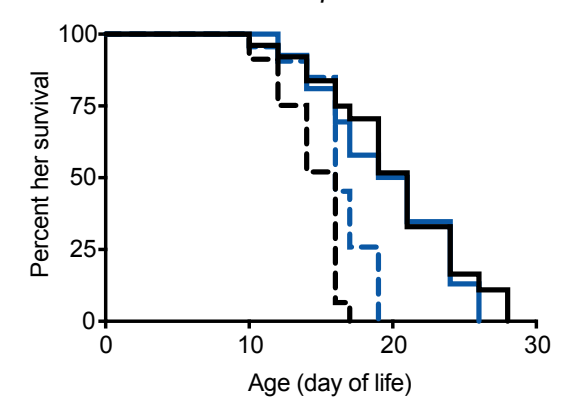

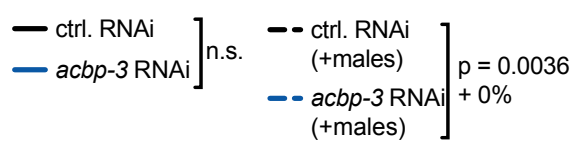

j

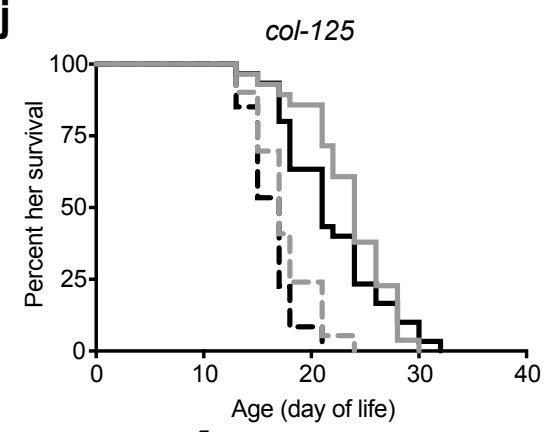

$\left.\begin{array}{cc}- \text { ctrl. RNAi } \\ - \text { col-43 RNAi }]+14 \% \text { n.s. } & - \text { ctrl. RNAi } \\ & (+ \text { males }) \\ -- & \text { col-43 RNAi } \\ & (+ \text { males })\end{array}\right] p=0.0243$ b

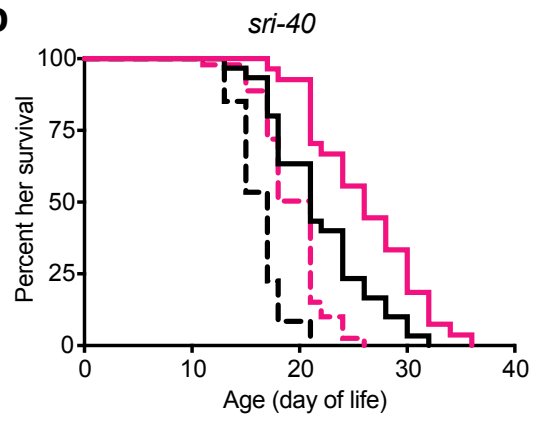

$\left.\left.\begin{array}{cc}\text { - ctrl. RNAi } \\ \text { sri-40 RNAi }\end{array}\right] \begin{array}{l}p=0.0031 \\ +24 \%\end{array} \quad \begin{array}{l}\text { ctrl. RNAi } \\ \text { (+males) } \\ -\begin{array}{l}\text { sri-40 RNAi } \\ \text { (+males) }\end{array}\end{array}\right] \begin{aligned} & p<0.0001 \\ & +23.5 \%\end{aligned}$

e

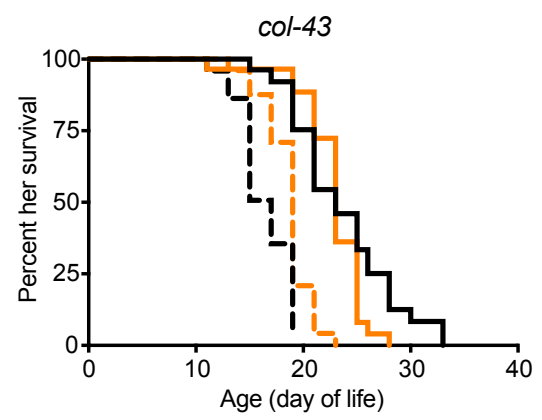

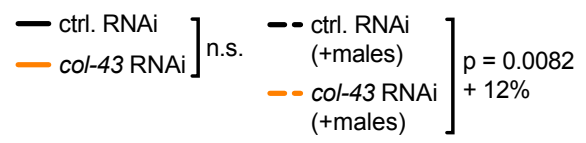

h
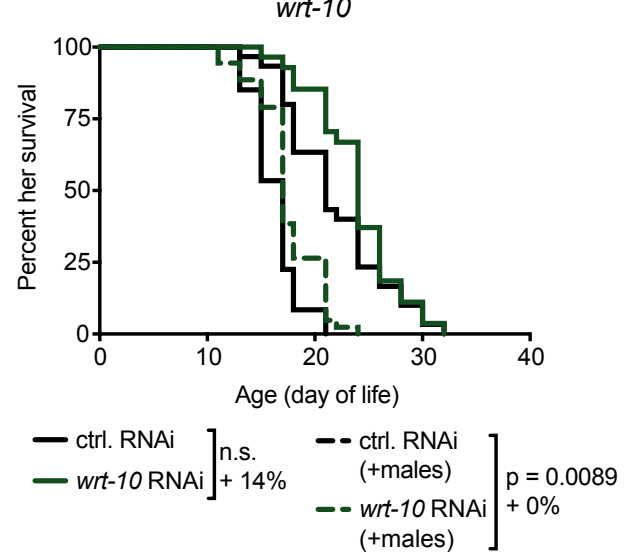

k

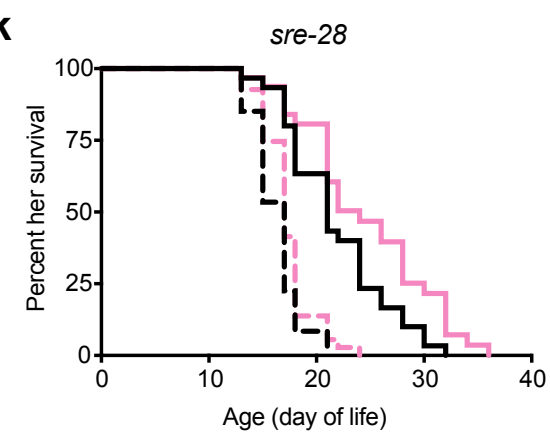

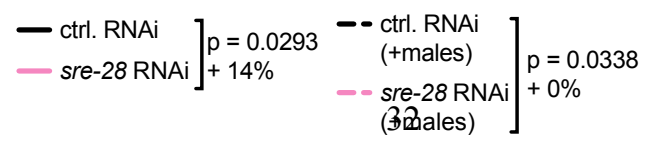




\section{Extended Data Figure 2: RNAi screen results}

(a) A summary of the results of the RNAi-based screen to identify functionally important maleinduced gene expression changes. The colors of the circles indicate the change in median lifespan relative to control, empty vector RNAi. The sizes of the circles indicate the $p$-values calculated using Mantel-Cox log ranking. Hits from the screen are shown with red gene name labels. Genes are ordered from smallest to largest change in median lifespan in the presence of males, using $p$ value to break ties. (b-k) The Kaplan-Meier survival curves of the screen hits $(p \leq 0.05$ when comparing control RNAi versus gene of interest RNAi in the presence of males). Control RNAi treatment is in black and gene of interest RNAi treatment is plotted as colored lines. Hermaphrodite lifespans measured in the presence of males are shown as dashed lines. Several of the control RNAi curves are identical between panels because these genes were tested together in a group of blinded conditions with a single control RNAi condition. For the screen, 23-59 wild-type hermaphrodites were tested. For each lifespan curve comparison, the $p$-value (Mantel-Cox log ranking) and percent change in median lifespan are shown. See Supplementary Table 1 for extended statistics as well as the groupings of the genes tested in the screen. 
bioRxiv preprint doi: https://doi.org/10.1101/2020.06.30.181008; this version posted July 1, 2020. The copyright holder for this preprint (which was not certified by peer review) is the author/funder, who has granted bioRxiv a license to display the preprint in perpetuity. It is made

\section{Extended Data Figure 3}

a

Pearson's correlations

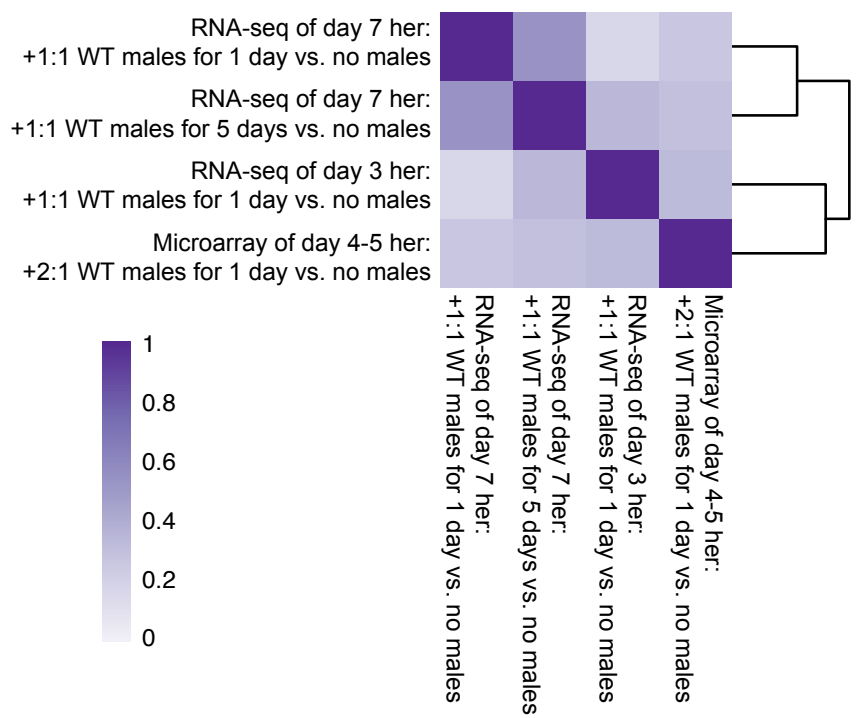

C

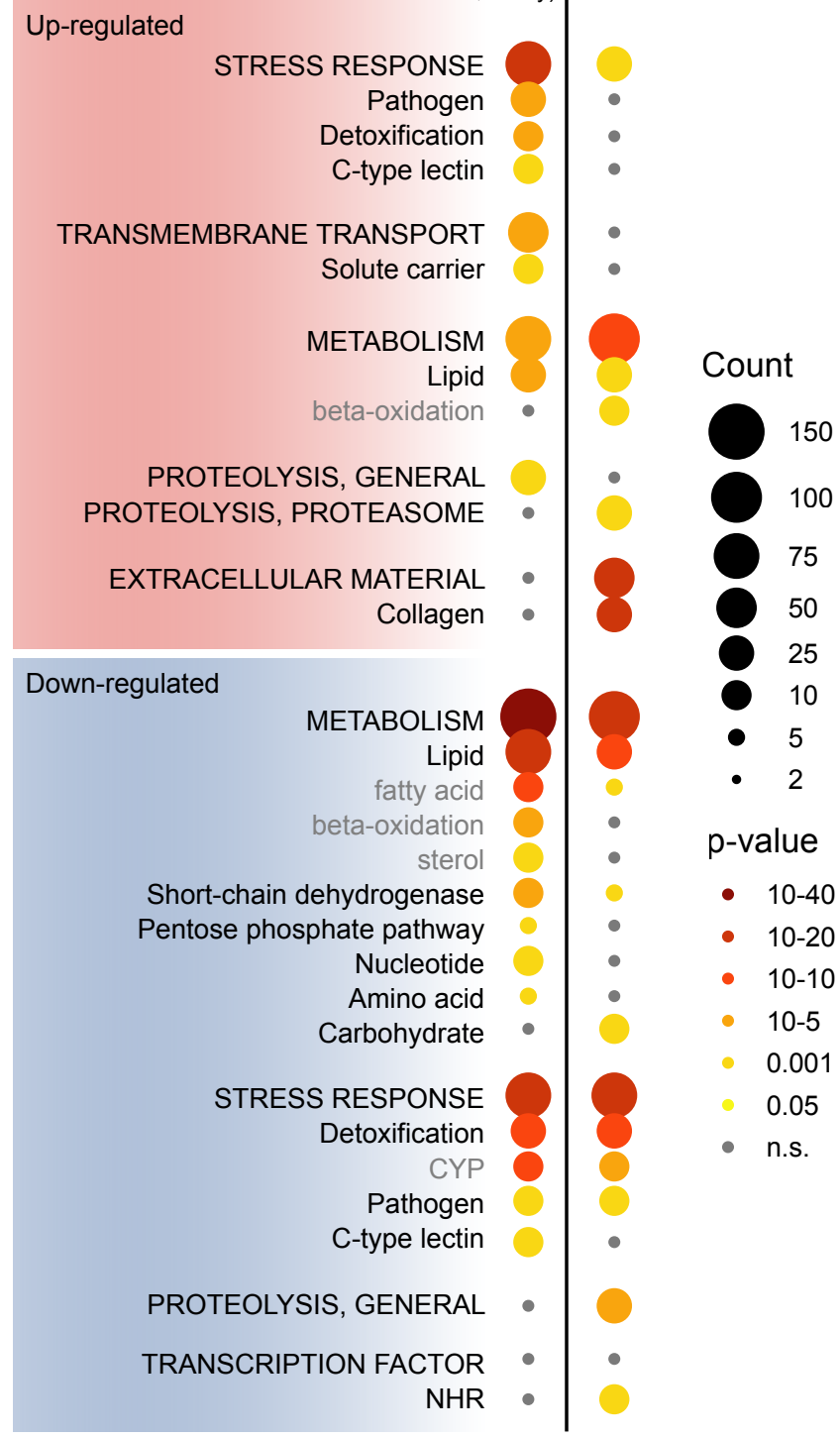

b

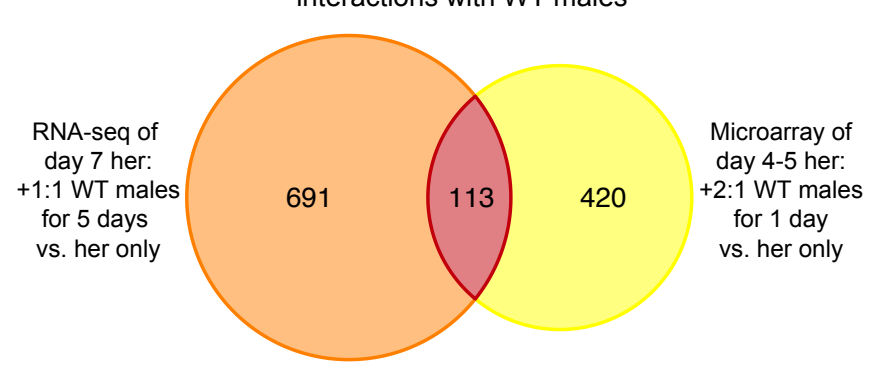

Genes down-regulated by sexual interactions with WT males

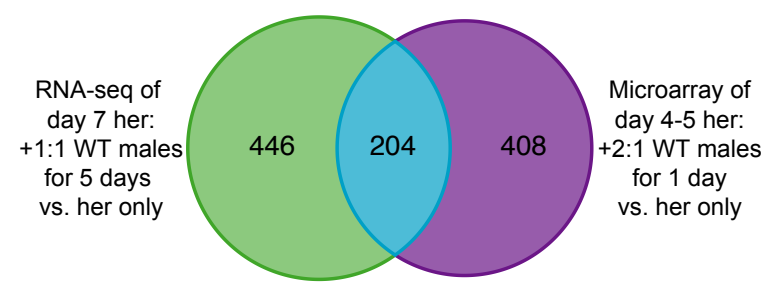

d

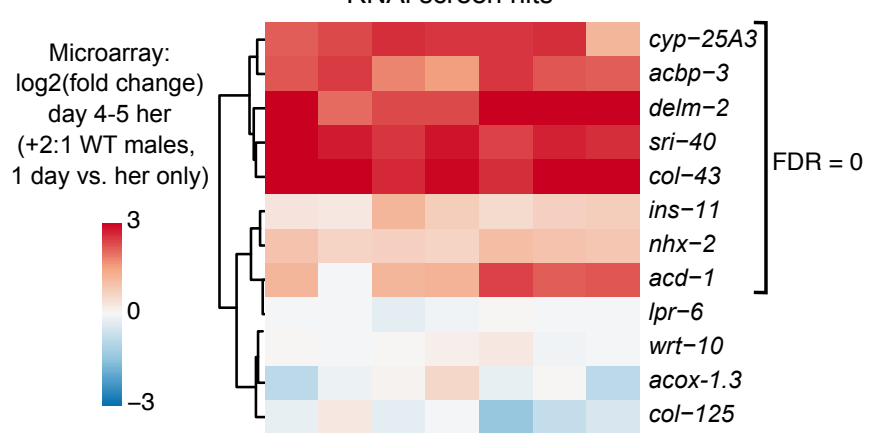

e
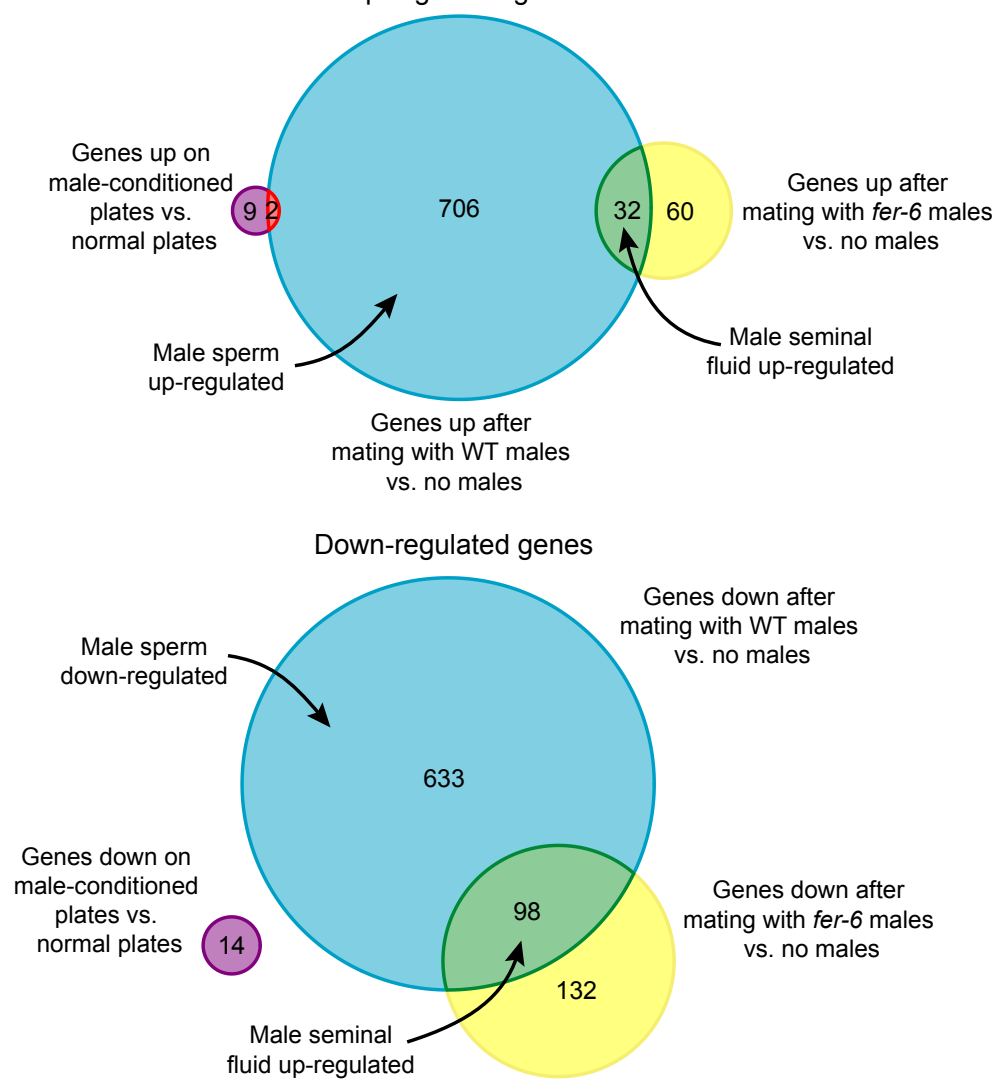


\section{Extended Data Figure 3: Comparison of RNA-seq and microarray results}

The RNA-seq and microarray experiments were performed by two different researchers at different institutes using different experimental setups (e.g. the ratio of males to hermaphrodites and the age of the hermaphrodites) and different alleles of $g l p-1$. For complete details, see the Materials and Methods. (a) Pearson's correlation scores comparing the wild-type male-induced fold-change in hermaphrodite gene expression detected in the $g l p-1$ hermaphrodite RNA-seq datasets and the $g l p-1$ hermaphrodite microarray dataset. (b) Venn diagrams of the wild-type maleinduced differentially expressed hermaphrodite genes detected by microarray and RNA-seq. The number of overlapping differentially expressed genes is higher than expected by chance (upregulated: $p=6.15 \times 10^{-21}$, downregulated: $p=1.69 \times 10^{-93}$, hypergeometric test). (c) Enriched gene categories in the hermaphrodite genes that are upregulated (red) and downregulated (blue) by wild-type males in the microarray and the RNA-seq results. The number of differentially expressed genes in each gene set is indicated by the size of the circle and the significance of the enrichment by the color of the circle. Gene annotations are nested with the broadest categories listed in all capital letters and the middle categories listed with the first letter capitalized, and the most specific categories in grey. The gene category enrichment for all datasets, including those not shown here, can be found in Supplementary Tables 3 and 9. (d) A heatmap of the microarray results from $g l p-1$ hermaphrodites mated with WT males for one day. The genes that we identified as functionally important for male-induced demise (Fig. 1i and Extended Data Fig. 2) are shown, including ins-11 $11^{1}$. The majority of genes that were differentially expressed in our RNA-seq and identified as functionally important in our screen were also differentially expressed in the microarray experiment. The four genes that were not significantly up-regulated under the different experimental conditions used for the microarray were not investigated further. sre-28 was not detected in the microarray. (e) Venn diagrams of the significantly up- and down-regulated hermaphrodite genes detected by microarray following one day of interacting (mating) with WT males or fer-6(hc6) sperm-less males (blue and yellow, respectively) or five days on maleconditioned plates (i.e. male secreted compounds and pheromones, purple). A complete list of all differentially expressed genes from the RNA-seq and microarrays can be found in Supplementary Tables 2, 6, 7, and 8 . 
bioRxiv preprint doi: https://doi.org/10.1101/2020.06.30.181008; this version posted July 1, 2020. The copyright holder for this preprint (which was not certified by peer review) is the author/funder, who has granted bioRxiv a license to display the preprint in perpetuity. It is made available under aCC-BY-NC-ND 4.0 International license.

\section{Extended Data Figure 4}

a

Pairwise alignment:

delm-2 targeting RNAi and acd-1 and delm-1 paralogs

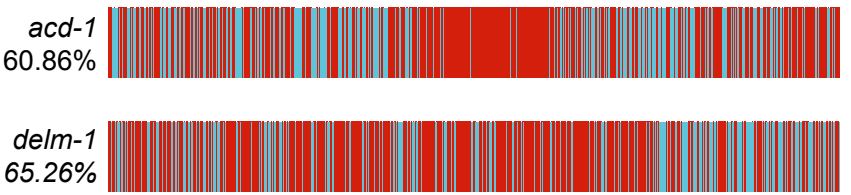

delm-2 RNAi sequence

1038

Identical nucleotide

Unique nucleotide

C

acd-1

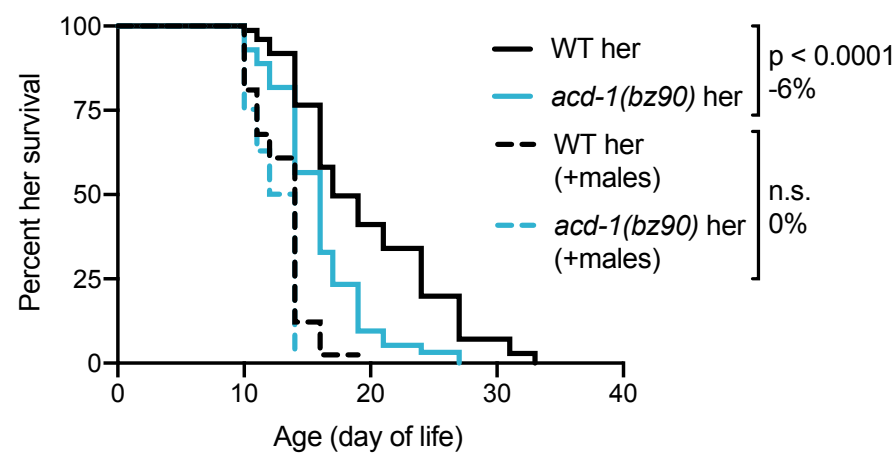

e

delm-1

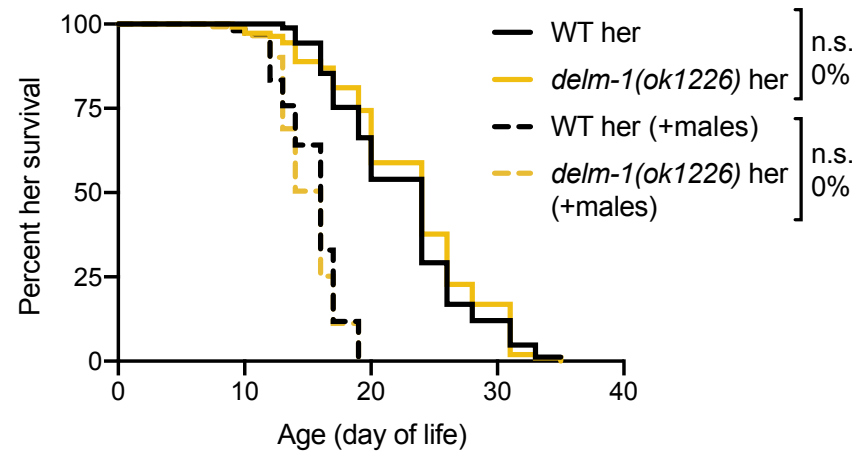

b

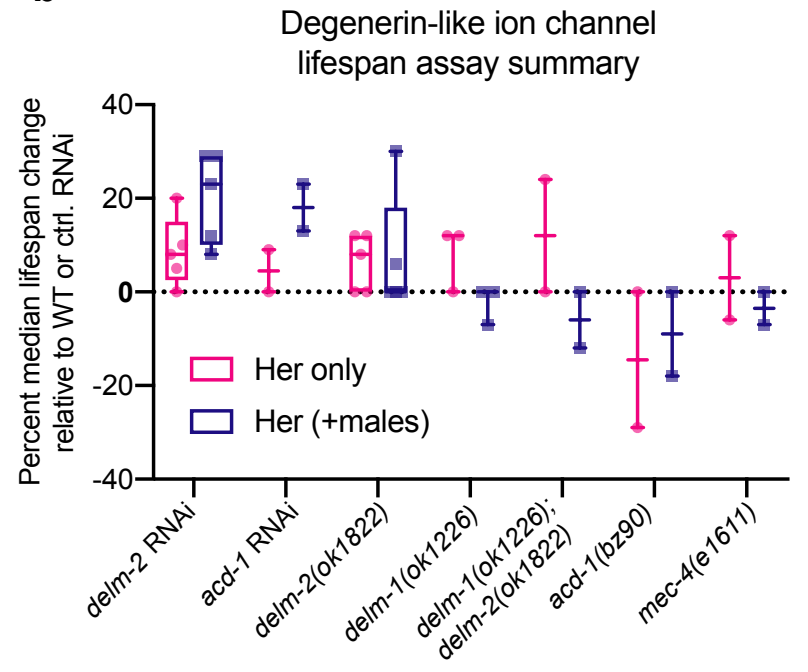

d

delm-2

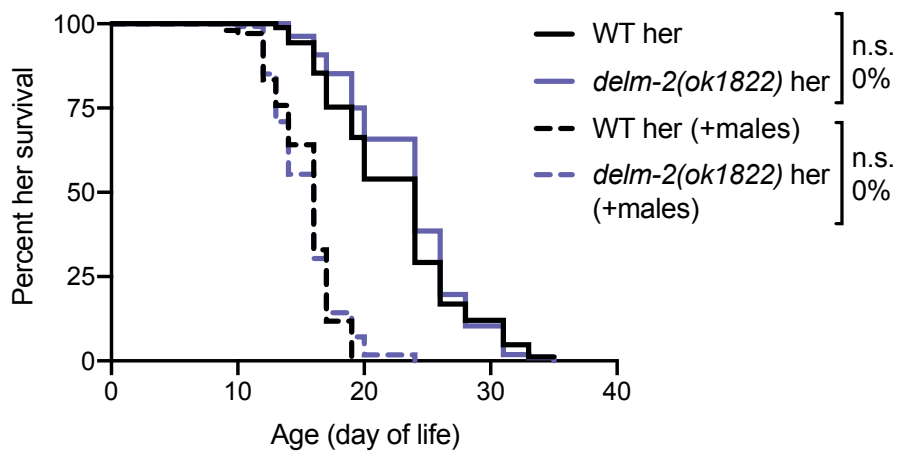

f

delm-1, delm-2

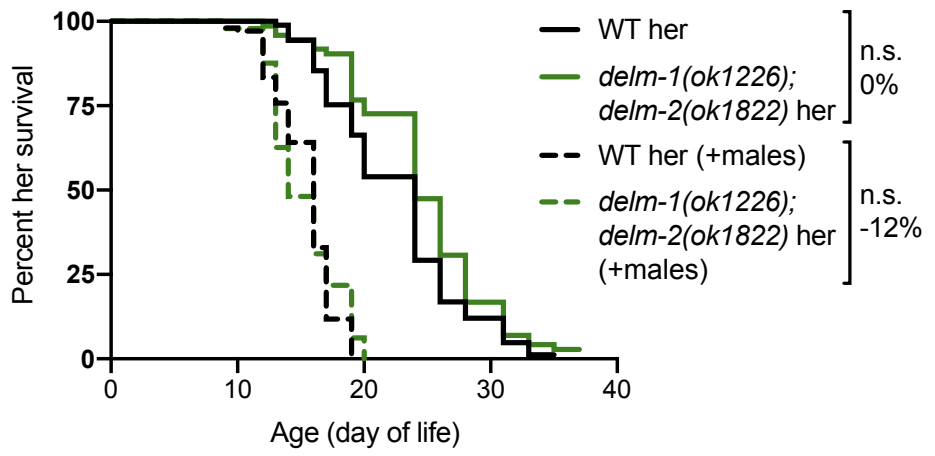




\section{Extended Data Figure 4: Degenerin-like ion channel lifespan assays}

(a) DNA sequence alignment of the delm-2 RNAi targeting sequence from the Ahringer RNAi library ${ }^{35}$ with the aligning portions of the delm-1 and acd-1 unspliced transcripts. (b) A summary of the male-induced demise lifespan assay results testing the role of the degenerin-like ion channel paralogs acd-1, delm-1, and delm-2. Lifespan data (median lifespan change relative to control) for each biological replicate are shown as individual data points and summarized with a box plot. Box plot whiskers show minimum and maximum and the line in the box indicates the median of the biological replicates. (c-f) Loss of function mutations in acd-1, delm-1, or delm-2 alone (panels ce) were not sufficient to extend hermaphrodite lifespan in the presence or absence of males, nor did delm-1, delm-2 double mutation (panel f). We note that RNAi targeting either delm-2 or acd1 is sufficient to extend hermaphrodite lifespan (Fig. 2e and Fig. 4d, g). 89-138 hermaphrodites were tested in panels b-f. Lifespans are plotted as Kaplan Meier survival curves and $p$-values calculated by Mantel-Cox log ranking. Percent change in median lifespan compared to control is shown in panels c-f. See Supplementary Table 1 for extended statistics. 
bioRxiv preprint doi: https://doi.org/10.1101/2020.06.30181008; this version posted July 1, 2020. The copyright holder for this preprint (which was not certified by peer review) is the author/funder, who has granted bioRxiv a license to display the preprint in perpetuity. It is made available under aCC-BY-NC-ND 4.0 International license.

\section{Extended Data Figure 5}

a

Male-conditioned plates

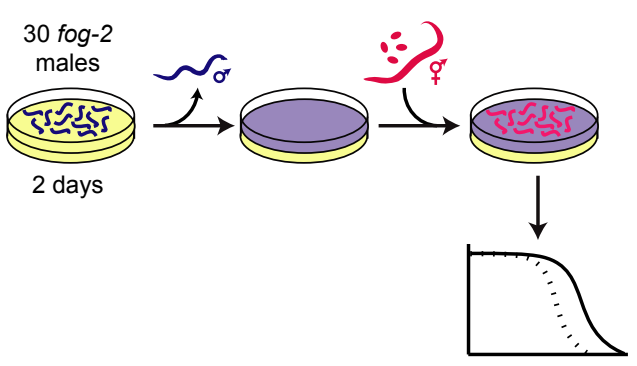

C

$c d r-1$ RNAi

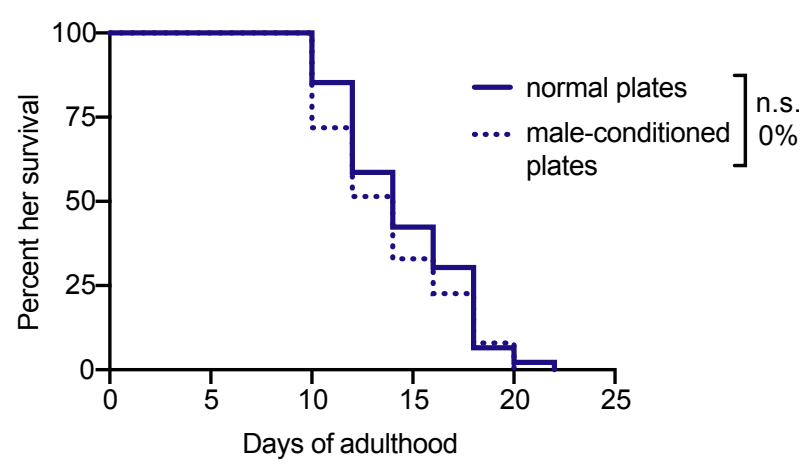

e

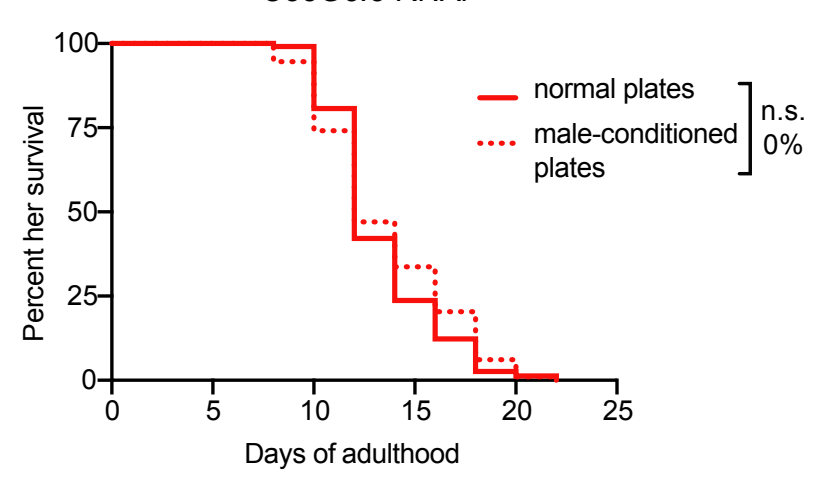

b

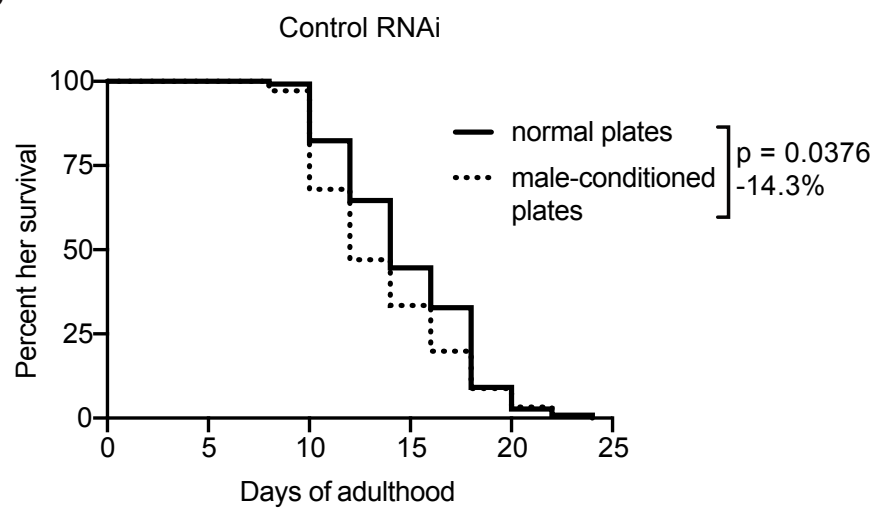

d

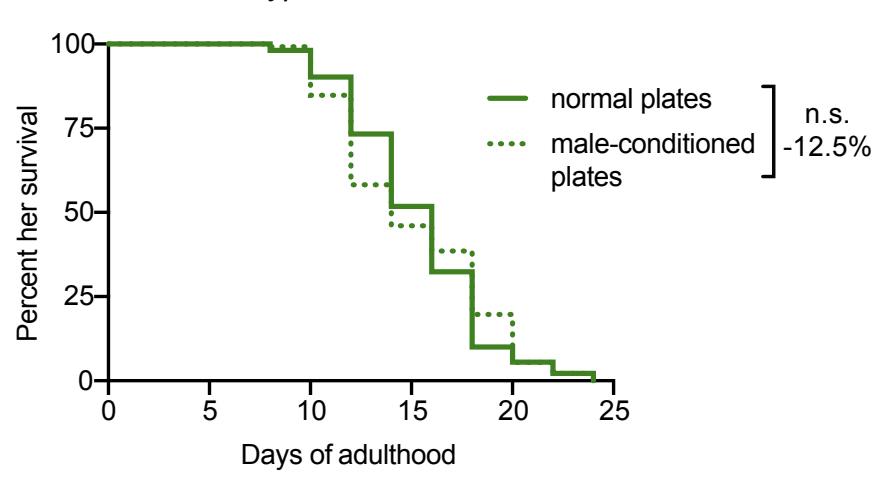

f

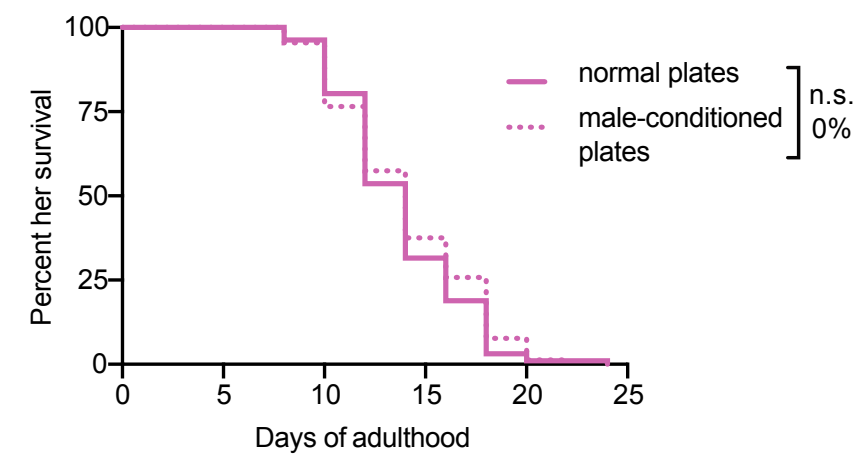




\section{Extended Data Figure 5: Male-conditioned plate screen results}

(a) A scheme describing the experimental set up for the male-conditioned plate lifespan assays. For complete details, see the Materials and Methods. (b-f) The lifespans of hermaphrodites fed control empty vector RNAi (b) or RNAi against the male-conditioned plate induced genes $c d r-1$ (c), cyp-35D1 (d), C33G8.3 (e), or hmit-1.1 (f). Hermaphrodite worms were either exposed to media conditioned by $30 \mathrm{fog}$-2(q71) males for 2 days (removed prior to placing the hermaphrodites on the plate) (dotted lines) or were placed on normal plates (solid lines). Results are plotted as Kaplan-Meier survival curves and $p$-values calculated using Mantel-Cox log ranking. Percent change in median lifespan compared to normal plates is shown in each panel. 118-132 hermaphrodites were tested in each condition. See Supplementary Table 1 for a complete list of all lifespan assay results. 
bioRxiv preprint doi: https://doi.org/10.1101/2020.06.30.181008; this version posted July 1, 2020. The copyright holder for this preprint (which was not certified by peer review) is the author/funder, who has granted bioRxiv a license to display the preprint in perpetuity. It is made Extended Data Figure 6 available under aCC-BY-NC-ND 4.0 International license.

a
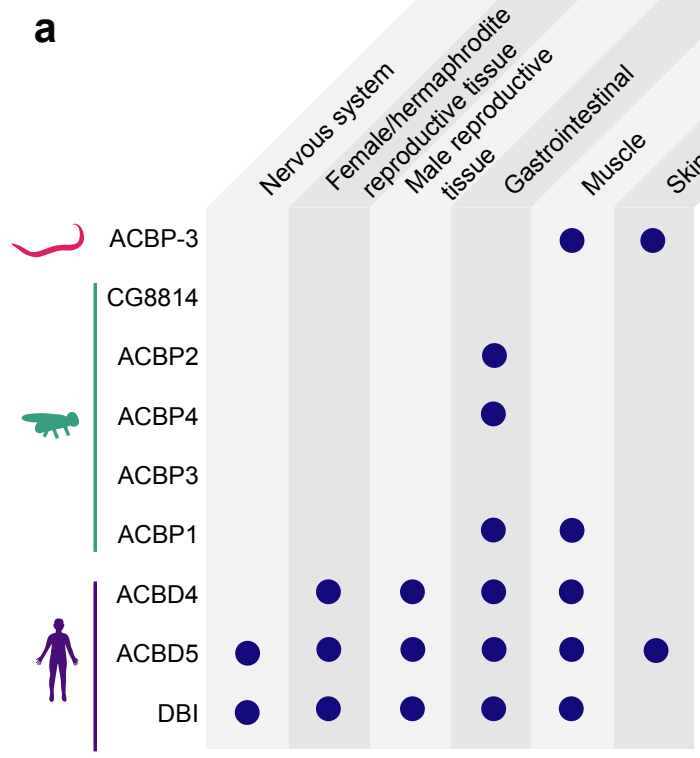

b

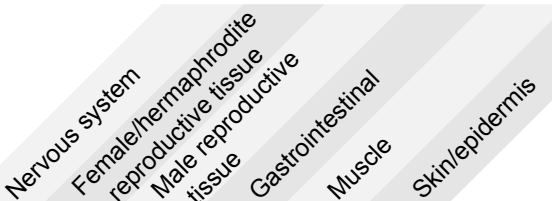

$\left.\circlearrowright\right|_{\text {DELM-2 }} ^{\text {ACD-1 }}$

$\mid$\begin{tabular}{r|} 
PPK28 \\
PPK14 \\
PPK12 \\
PPK8 \\
PPK21
\end{tabular}

| $\begin{array}{lll}\text { ASIC5 } & & \\ \text { ASIC2 } & 0 & 0 \\ \text { ASIC1 } & 0 \\ \text { ASIC4 } & 0 \\ \text { ASIC3 } & 0 & 0\end{array}$
C

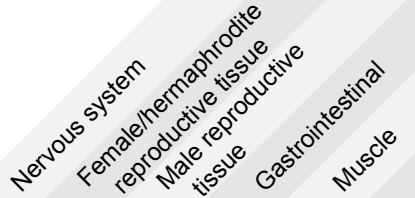

COL-43

COL4A
VKG

CG42342

MP

COL21A1

COL24A1

11

C1QTNF9B

COL4A1

d

HMIT-1.1

$\mid \begin{array}{r}\text { TRET1-2 } \\ \text { TRET1-1 } \\ \text { GLUT1 } \\ \text { CG6484 } \\ \text { SUT1 }\end{array}$

$\mid \begin{aligned} & \mathrm{SLC} 2 \mathrm{~A} 13 \\ & \mathrm{SLC} 2 \mathrm{~A} 12 \\ & \mathrm{SLC} 2 \mathrm{~A} 10 \\ & \mathrm{SLC} 2 \mathrm{~A} 2 \\ & \mathrm{SLC} 2 \mathrm{~A} 3\end{aligned}$

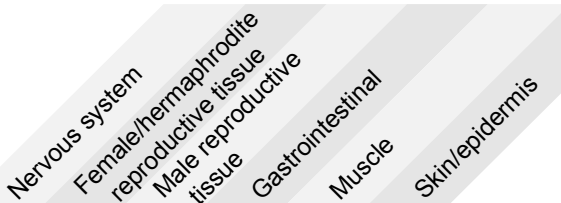

e

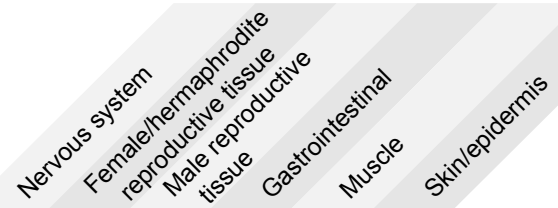

\begin{tabular}{|r} 
NHX-2 \\
NHE2 \\
NHE1 \\
NHE3
\end{tabular} \mid $\begin{aligned} & \text { SLC9A2 } \\
& \text { SLC9A3 } \\
& \text { SLC9A4 } \\
& \text { SLC9A1 } \\
& \text { SLC9A5 }\end{aligned}$

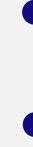

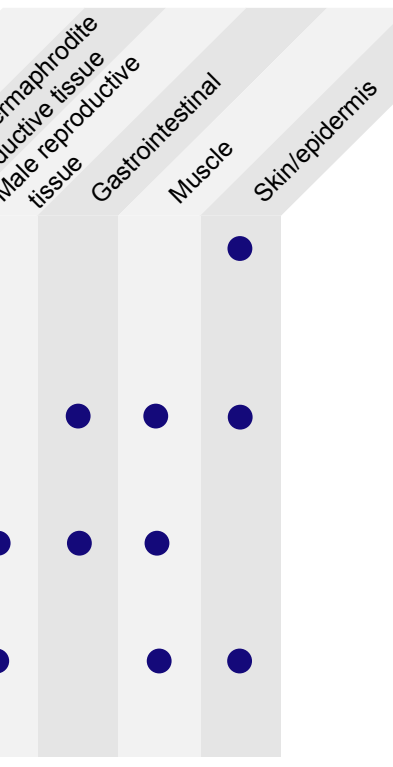

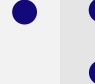

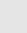




\section{Extended Data Figure 6: Conservation of male-induced demise genes}

The top five BLASTp hits in Drosophila melanogaster and humans for the C. elegans maleinduced demise genes acbp-3 (a), acd-1 and delm-2 (b), col-43 (c), hmit-1.1 (d), and nhx-2 (e). The tissue-specific protein expression is indicated for each ortholog in $C$. elegans (www.wormbase.org, version WS275), Drosophila (www.flybase.org, version 2020_01), and humans (www.proteinatlas.com ${ }^{46,47}$ ). A portion of these data are also presented in Figure $2 \mathrm{~g}-\mathrm{i}$. 
bioRxiv preprint doi: https://doi.org/10.1101/2020.06.30.181008; this version posted July 1, 2020. The copyright holder for this preprint (which

was not certified by peer review) is the author/funder, who has granted bioRxiv a license to display the preprint in perpetuity. It is made available under aCC-BY-NC-ND 4.0 International license.

\section{Extended Data Figure 7}

a Transcription factor binding enrichment

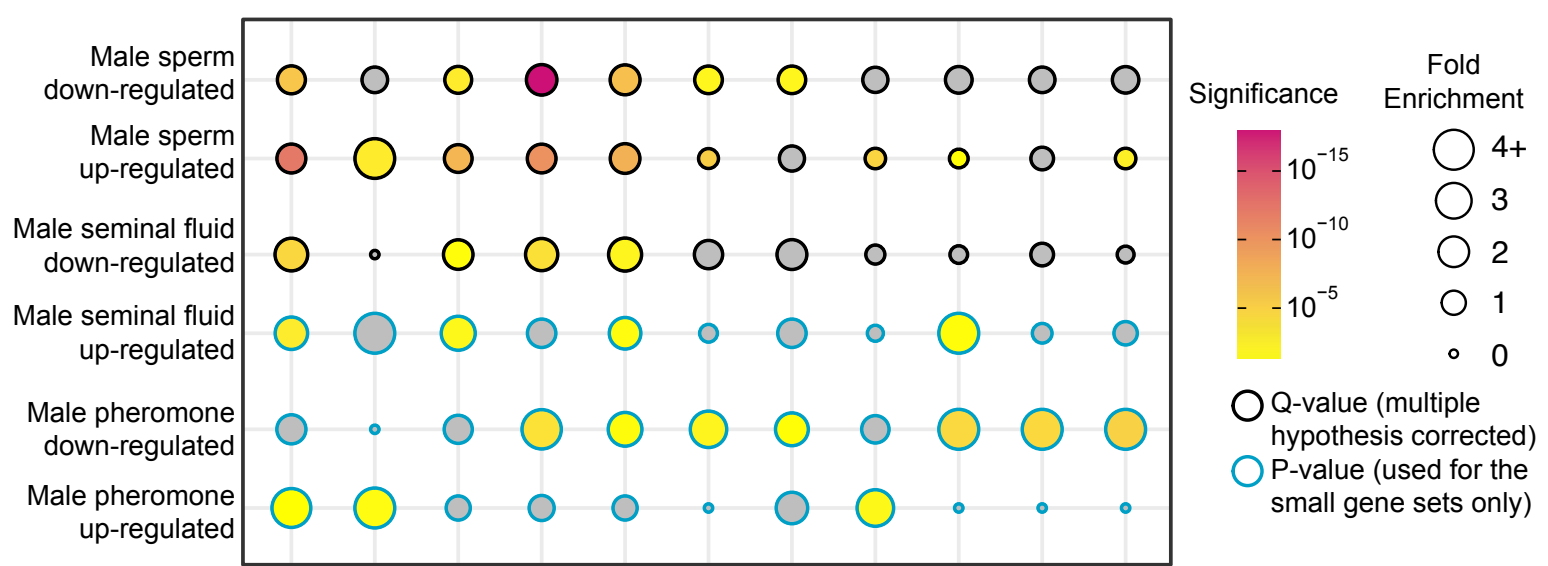

b

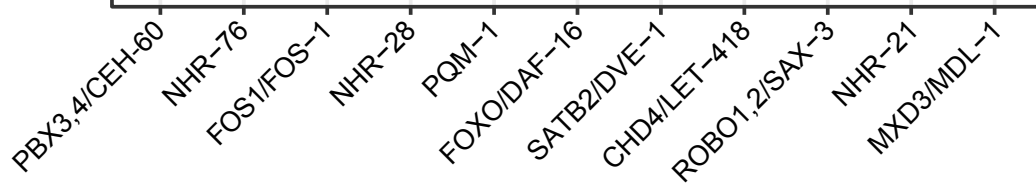

ELT-3 motif enrichment

Male sperm down-regulated
Male sperm up-regulated
Male seminal fluid down-regulated
Male seminal fluid up-regulated
Male pheromone down-regulated
Male pheromone up-regulated
PQM-1 motif enrichment

\section{ACTGATAAGA}

$<0.0001$

$<0.0001$

$<0.0001$

0.0655

n.s.

0.0060

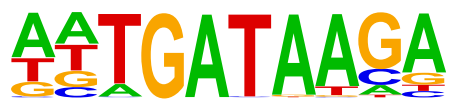

$<0.0001$

$<0.0001$

$<0.0001$

n.s.

n.s.

0.0549 


\section{Extended Data Figure 7: Transcription Factor Binding Enrichment}

(a) The enrichments of selected transcription factor binding peaks within $2 \mathrm{~kb}+/-$ the transcription start site of the male sperm, seminal fluid, and pheromone downregulated and upregulated genes are shown. Fold enrichment at the gene set of interest compared to genome-wide is indicated by the size of each circle and Q-value (false discovery corrected $p$-value) or $p$-value by the color of each circle. A complete list of the transcription factor binding enrichment is presented in Supplementary Table 10. (b) Enrichment of "known" transcription factor binding motifs within $300 \mathrm{bp}+/-$ the transcription start site of the differentially expressed genes. The Bonferronicorrected $p$-values for the motif enrichment for each gene set is shown. 


\section{Extended Data Figure 8}

\begin{tabular}{|c|c|c|c|}
\hline & $\begin{array}{l}\text { Her } \\
\text { only }\end{array}$ & $\begin{array}{c}\text { Her } \\
(+ \text { males })\end{array}$ & \multirow{5}{*}{$100 \%$} \\
\hline WT her on $1: 1$ acbp-3 and delm-2 RNAi & $20 \%$ & $47 \%$ & \\
\hline WT her on col-43 RNAi & $3 \%$ & $22 \%$ & \\
\hline acd-1 her & $-15 \%$ & $-9 \%$ & \\
\hline WT her on acd-1 RNAi & $5 \%$ & $18 \%$ & \\
\hline WT her on delm-2 RNAi & $9 \%$ & $20 \%$ & \multirow{4}{*}{$0 \%$} \\
\hline WT her on acbp-3 RNAi & $6 \%$ & $11 \%$ & \\
\hline WT her on utx-1 RNAi & $14 \%$ & $15 \%$ & \\
\hline WT her on sri-40 RNAi & $11 \%$ & $13 \%$ & \\
\hline WT her on $n h x-2 \mathrm{RNAi}$ & $16 \%$ & $15 \%$ & \multirow{2}{*}{$-100 \%$} \\
\hline nuo-6(qm200) her & $69 \%$ & $48 \%$ & \\
\hline WT her on col-125 RNAi & $12 \%$ & $0 \%$ & \\
\hline daf-2(e1370) her & $133 \%$ & $12 \%$ & \\
\hline
\end{tabular}




\section{Extended Data Figure 8: Male-induced demise and longevity}

A summary of the percent loss of lifespan in the presence of males in the mutant or RNAi knockdown compared to wild-type. Positive values indicate that the genetic manipulation resulted in greater protection from male-induced demise whereas negative values indicate hypersensitivity to male-induced demise. The daf-2(e1370) results were published previously ${ }^{1}$. All other lifespan results are from this manuscript (see Supplementary Table 1 and Supplementary Table 11). 
Supplementary Tables

Supplementary Table 1: Lifespan assay results

Supplementary Table 2: DESeq2 calculated RNA-seq differential expression after removal of male-enriched transcripts

Supplementary Table 3: Gene set enrichment of the RNA-seq differentially expressed genes (after removal of male-enriched transcripts)

Supplementary Table 4: DESeq2 calculated RNA-seq differential expression

Supplementary Table 5: Gene set enrichment of the RNA-seq differentially expressed genes

Supplementary Table 6: Microarray SAM results for WT male-mated hermaphrodites

Supplementary Table 7: Microarray SAM results for spermless (fer-6) male-mated hermaphrodites

Supplementary Table 8: Microarray SAM results for male-conditioned plate exposed hermaphrodites

Supplementary Table 9: Gene set enrichment of the microarray differentially expressed genes

Supplementary Table 10: Transcription factor ChIP-seq enrichment

Supplementary Table 11: Comparison of lifespans in the presence and absence of males 


\section{References}

1 Maures, T. J. et al. Males shorten the life span of C. elegans hermaphrodites via secreted compounds. Science 343, 541-544, doi:10.1126/science. 1244160 (2014).

2 Shi, C. \& Murphy, C. T. Mating induces shrinking and death in Caenorhabditis mothers. Science 343, 536-540, doi:10.1126/science.1242958 (2014).

3 Gems, D. \& Riddle, D. L. Longevity in Caenorhabditis elegans reduced by mating but not gamete production. Nature 379, 723-725, doi:10.1038/379723a0 (1996).

4 Fowler, K. \& Partridge, L. A Cost of Mating in Female Fruit-Flies. Nature 338, 760-761, doi:DOI 10.1038/338760a0 (1989).

5 Gendron, C. M. et al. Drosophila life span and physiology are modulated by sexual perception and reward. Science 343, 544-548, doi:10.1126/science.1243339 (2014).

6 Garratt, M., Kee, A. J., Palme, R. \& Brooks, R. C. Male Presence can Increase Body Mass and Induce a Stress-Response in Female Mice Independent of Costs of Offspring Production. Sci Rep 6, 23538, doi:10.1038/srep23538 (2016).

7 Wigby, S. \& Chapman, T. Sex peptide causes mating costs in female Drosophila melanogaster. Curr Biol 15, 316-321, doi:10.1016/j.cub.2005.01.051 (2005).

8 Palopoli, M. F. et al. Natural and experimental evolution of sexual conflict within Caenorhabditis nematodes. BMC Evol Biol 15, 93, doi:10.1186/s12862-015-0377-2 (2015).

9 Garratt, M., Try, H., Smiley, K. O., Grattan, D. R. \& Brooks, R. C. Mating in the absence of fertilization promotes a growth-reproduction versus lifespan trade-off in female mice. Proc Natl Acad Sci U S A, doi:10.1073/pnas.2003159117 (2020).

10 Ludewig, A. H. et al. An excreted small molecule promotes C. elegans reproductive development and aging. Nat Chem Biol 15, 838-845, doi:10.1038/s41589-019-0321-7 (2019).

11 Shi, C., Runnels, A. M. \& Murphy, C. T. Mating and male pheromone kill Caenorhabditis males through distinct mechanisms. Elife 6, doi:10.7554/eLife.23493 (2017).

12 Shi, C., Booth, L. N. \& Murphy, C. T. Insulin-like peptides and the mTOR-TFEB pathway protect Caenorhabditis elegans hermaphrodites from mating-induced death. Elife 8, doi:10.7554/eLife.46413 (2019).

13 Booth, L. N., Maures, T. J., Yeo, R. W., Tantilert, C. \& Brunet, A. Self-sperm induce resistance to the detrimental effects of sexual encounters with males in hermaphroditic nematodes. Elife 8, doi:10.7554/eLife.46418 (2019).

14 Bazopoulou, D. et al. Developmental ROS individualizes organismal stress resistance and lifespan. Nature 576, 301-305, doi:10.1038/s41586-019-1814-y (2019).

15 Aprison, E. Z. \& Ruvinsky, I. Sexually Antagonistic Male Signals Manipulate Germline and Soma of C. elegans Hermaphrodites. Curr Biol 26, 2827-2833, doi:10.1016/j.cub.2016.08.024 (2016).

16 Priess, J. R., Schnabel, H. \& Schnabel, R. The glp-1 locus and cellular interactions in early C. elegans embryos. Cell 51, 601-611 (1987).

17 Argon, Y. \& Ward, S. Caenorhabditis elegans fertilization-defective mutants with abnormal sperm. Genetics 96, 413-433 (1980).

18 Lee, S. H. et al. Modulatory upregulation of an insulin peptide gene by different pathogens in C. elegans. Virulence 9, 648-658, doi:10.1080/21505594.2018.1433969 (2018).

19 Han, L. et al. Two novel DEG/ENaC channel subunits expressed in glia are needed for nosetouch sensitivity in Caenorhabditis elegans. $J$ Neurosci 33, 936-949, doi:10.1523/JNEUROSCI.2749-12.2013 (2013). 
20 Wang, Y. et al. A glial DEG/ENaC channel functions with neuronal channel DEG-1 to mediate specific sensory functions in C. elegans. EMBO J 27, 2388-2399, doi:10.1038/emboj.2008.161 (2008).

21 Jin, C. et al. Histone demethylase UTX-1 regulates C. elegans life span by targeting the insulin/IGF-1 signaling pathway. Cell Metab 14, 161-172, doi:10.1016/j.cmet.2011.07.001 (2011).

22 Maures, T. J., Greer, E. L., Hauswirth, A. G. \& Brunet, A. The H3K27 demethylase UTX-1 regulates C. elegans lifespan in a germline-independent, insulin-dependent manner. Aging Cell 10, 980-990, doi:10.1111/j.1474-9726.2011.00738.x (2011).

23 Kenyon, C., Chang, J., Gensch, E., Rudner, A. \& Tabtiang, R. A C. elegans mutant that lives twice as long as wild type. Nature 366, 461-464, doi:10.1038/366461a0 (1993).

24 Kimura, K. D., Tissenbaum, H. A., Liu, Y. \& Ruvkun, G. daf-2, an insulin receptor-like gene that regulates longevity and diapause in Caenorhabditis elegans. Science 277, 942-946, doi:10.1126/science.277.5328.942 (1997).

25 Arantes-Oliveira, N., Apfeld, J., Dillin, A. \& Kenyon, C. Regulation of life-span by germ-line stem cells in Caenorhabditis elegans. Science 295, 502-505, doi:10.1126/science.1065768 (2002).

26 Feng, J., Bussiere, F. \& Hekimi, S. Mitochondrial electron transport is a key determinant of life span in Caenorhabditis elegans. Dev Cell 1, 633-644, doi:10.1016/s1534-5807(01)000715 (2001).

27 Yang, W. \& Hekimi, S. Two modes of mitochondrial dysfunction lead independently to lifespan extension in Caenorhabditis elegans. Aging Cell 9, 433-447, doi:10.1111/j.14749726.2010.00571.x (2010).

28 Senchuk, M. M. et al. Activation of DAF-16/FOXO by reactive oxygen species contributes to longevity in long-lived mitochondrial mutants in Caenorhabditis elegans. PLoS Genet 14, e1007268, doi:10.1371/journal.pgen.1007268 (2018).

29 Consortium, C. e. D. M. large-scale screening for targeted knockouts in the Caenorhabditis elegans genome. G3 (Bethesda) 2, 1415-1425, doi:10.1534/g3.112.003830 (2012).

30 Dobin, A. et al. STAR: ultrafast universal RNA-seq aligner. Bioinformatics 29, 15-21, doi:10.1093/bioinformatics/bts635 (2013).

31 Love, M. I., Huber, W. \& Anders, S. Moderated estimation of fold change and dispersion for RNA-seq data with DESeq2. Genome Biol 15, 550, doi:10.1186/s13059-014-0550-8 (2014).

32 Huber, W. et al. Orchestrating high-throughput genomic analysis with Bioconductor. Nat Methods 12, 115-121, doi:10.1038/nmeth.3252 (2015).

33 Holdorf, A. D. et al. WormCat: An Online Tool for Annotation and Visualization of Caenorhabditis elegans Genome-Scale Data. Genetics 214, 279-294, doi:10.1534/genetics.119.302919 (2020).

34 Lucanic, M. et al. Impact of genetic background and experimental reproducibility on identifying chemical compounds with robust longevity effects. Nat Commun 8, 14256, doi:10.1038/ncomms14256 (2017).

35 Kamath, R. S. et al. Systematic functional analysis of the Caenorhabditis elegans genome using RNAi. Nature 421, 231-237, doi:10.1038/nature01278 (2003).

36 Rual, J. F. et al. Toward improving Caenorhabditis elegans phenome mapping with an ORFeome-based RNAi library. Genome Res 14, 2162-2168, doi:10.1101/gr.2505604 (2004). 
37 Tusher, V. G., Tibshirani, R. \& Chu, G. Significance analysis of microarrays applied to the ionizing radiation response. Proc Natl Acad Sci $U$ S A 98, 5116-5121, doi:10.1073/pnas.091062498 (2001).

38 Sievers, F. et al. Fast, scalable generation of high-quality protein multiple sequence alignments using Clustal Omega. Mol Syst Biol 7, 539, doi:10.1038/msb.2011.75 (2011).

39 Waterhouse, A. M., Procter, J. B., Martin, D. M., Clamp, M. \& Barton, G. J. Jalview Version 2--a multiple sequence alignment editor and analysis workbench. Bioinformatics 25, 11891191, doi:10.1093/bioinformatics/btp033 (2009).

40 Oki, S. et al. ChIP-Atlas: a data-mining suite powered by full integration of public ChIP-seq data. EMBO Rep 19, doi:10.15252/embr.201846255 (2018).

41 Zhao, G. et al. Conserved Motifs and Prediction of Regulatory Modules in Caenorhabditis elegans. G3 (Bethesda) 2, 469-481, doi:10.1534/g3.111.001081 (2012).

42 Heinz, S. et al. Simple combinations of lineage-determining transcription factors prime cisregulatory elements required for macrophage and B cell identities. Mol Cell 38, 576-589, doi:10.1016/j.molcel.2010.05.004 (2010).

$43 \mathrm{Yu}$, G., Wang, L. G. \& He, Q. Y. ChIPseeker: an R/Bioconductor package for ChIP peak annotation, comparison and visualization. Bioinformatics 31, 2382-2383, doi:10.1093/bioinformatics/btv145 (2015).

44 Quinlan, A. R. \& Hall, I. M. BEDTools: a flexible suite of utilities for comparing genomic features. Bioinformatics 26, 841-842, doi:10.1093/bioinformatics/btq033 (2010).

45 McLeay, R. C. \& Bailey, T. L. Motif Enrichment Analysis: a unified framework and an evaluation on ChIP data. BMC Bioinformatics 11, 165, doi:10.1186/1471-2105-11-165 (2010).

46 Uhlen, M. et al. Proteomics. Tissue-based map of the human proteome. Science 347, 1260419, doi:10.1126/science.1260419 (2015).

47 Uhlen, M. et al. Towards a knowledge-based Human Protein Atlas. Nat Biotechnol 28, 12481250, doi:10.1038/nbt1210-1248 (2010).

48 Reinke, V. et al. A global profile of germline gene expression in C. elegans. Mol Cell 6, 605616, doi:10.1016/s1097-2765(00)00059-9 (2000). 